\title{
The Influence of Slide-Roll Ratio on the Extent of Micropitting Damage in Rolling-Sliding Contacts Pertinent to Gear Applications
}

\author{
Pawel Rycerz ${ }^{1}$ - Amir Kadiric ${ }^{1}[0$
}

Received: 29 October 2018 / Accepted: 23 April 2019 / Published online: 11 May 2019

(c) The Author(s) 2019

\begin{abstract}
Micropitting is a type of surface damage that occurs in rolling-sliding contacts operating under thin oil film, mixed lubrication conditions, such as those formed between meshing gear teeth. Like the more widely studied pitting damage, micropitting is caused by the general mechanism of rolling contact fatigue but, in contrast to pitting, it manifests itself through the formation of micropits on the local, roughness asperity level. Despite the fact that micropitting is increasingly becoming a major mode of gear failure, the relevant mechanisms are poorly understood and there are currently no established design criteria to assess the risk of micropitting occurrence in gears or other applications. This paper provides new understanding of the tribological mechanisms that drive the occurrence of micropitting damage and serves to inform the ongoing discussions on suitable design criteria in relation to the influence of contact slide-roll ratio (SRR) on micropitting. A triple-disc rolling contact fatigue rig is used to experimentally study the influence of the magnitude and direction of SRR on the progression of micropitting damage in samples made of case-carburised gear steel. The test conditions are closely controlled to isolate the influence of the variable of interest. In particular, any variation in bulk heating at different SRRs is eliminated so that tests are conducted at the same film thickness for all SRRs. The results show that increasing the magnitude of SRR increases the level of micropitting damage and that negative SRRs (i.e. the component where damage is being accumulated is slower) produce more micropitting than the equivalent positive SRRs. Measurements of elastohydrodynamic film thickness show that in the absence of bulk heating, increasing SRR does not cause a reduction in EHL film thickness and therefore this cannot be the reason for the increased micropitting at higher SRRs. Instead, we show that the main mechanism by which increase in SRR promotes micropitting is by increasing the number of micro-contact stress cycles experienced by roughness asperities during their passage through the rolling-sliding contact. Therefore, the asperity stress history should form the basis of any potential design criterion against micropitting.
\end{abstract}

Keywords Micropitting $\cdot$ Gears $\cdot$ Rolling contact fatigue $\cdot$ Stress history $\cdot$ Slide-roll ratio

\section{Introduction}

Micropitting is a type of surface damage that occurs in rolling-sliding contacts operating under mixed lubrication conditions associated with relatively thin oil films and high surface roughness. It is most commonly observed in gear teeth contacts, where it is a major design issue, but can also affect other machine components such as rolling bearings. Micropitting is caused by the general mechanism of rolling contact fatigue and manifests itself as numerous

Amir Kadiric

a.kadiric@imperial.ac.uk

1 Department of Mechanical Engineering, Tribology Group, Imperial College London, London SW7 2AZ, UK small pits and cracks on the surface of the affected component. It occurs through propagation of small, surfaceinitiated fatigue cracks, which grow at a shallow angle to the surface until a small segment of material detaches to form a micropit. The diameter and depth of such micropits is in the region of tens of microns. Damage is limited to the near-surface zone affected by the local stresses occurring due to surface roughness asperity contacts [1]. As such, micropitting differs from pitting which is characterized by pits of the order of overall contact size. However, both are fundamentally caused by the mechanism of rolling contact fatigue and involve the initiation and propagation of surface fatigue cracks. Although individual micropits are relatively small, micropitting damage typically spreads to cover a relatively large surface area and, if allowed to progress, 
eventually causes a significant loss of material from the load bearing surface, such as a gear tooth flank. Micropitting can therefore be thought of as a progressive fatigue wear process.

A review of relevant literature reveals multiple factors of influence on the onset and progression of micropitting. In particular, the relationship between the specific film thickness ( $\Lambda$ ratio) and micropitting severity has received substantial attention. In his studies on the influence of surface roughness on pitting behaviour, Dawson [2, 3] defined a parameter ' $D$ ' as a ratio of surface roughness to film thickness, equal to the reciprocal of what is now known as the specific film thickness or $\Lambda$ ratio. It is now well established that micropitting only occurs if the specific film thickness is low enough to facilitate severe asperity-to-asperity contact $[4,5]$. Other factors, including the magnitude and direction of sliding, level of Hertz contact pressure, material and running-in, have also been shown to have a significant influence on micropitting by several authors [6-12]. In addition to these experimental studies, a number of authors have attempted to predict the onset and progression of micropitting on gear teeth using numerical contact models $[8$, 13-15].

In a series of studies, Olver and co-workers $[4,5,16]$ showed that lubricant additives can affect the extent of micropitting by suppressing the wearing-in process, which in turn leads to an increased number of asperity stress cycles [5], and by changing the level of contact friction, which modifies tensile stresses and hence affects crack initiation and propagation [16]. These studies clearly indicated that the onset and progression of micropitting is highly dependent on the outcome of the continuous competition between surface fatigue and mild surface wear. The latter continuously modifies the surface roughness profiles and hence affects local fatigue damage accumulation. The importance of this competition on micropitting damage in gear teeth contact has recently been studied in detail by Morales-Espejel et al. [15] using a numerical approach that considers the concurrent effects of micropitting and mild wear on surface roughness evolution.

Despite this considerable body of work, the fundamental mechanisms of action of various factors which are known to influence micropitting remain poorly understood. Arguably the most significant amongst those is the influence of slide-roll ratio, where both the magnitude of sliding velocity and its direction are known to be important, but the exact mechanisms and suitable criteria to account for this influence are still debated. The outcome of this debate is of practical importance given the ongoing efforts in establishing suitable design criteria for prevention of micropitting $[18,19]$. Despite its apparent importance to gear reliability, there is currently no gear design standard aimed directly at prevention of micropitting. The lack of suitable design procedures means that micropitting failures may be frequently observed in what are otherwise perfectly well-designed gear sets. Some practical examples of micropitting in gears are illustrated in [17] amongst others. To address this issue, there are ongoing efforts within the international standards bodies to provide suitable guidance for assessing the risk of micropitting in gears, with two recent ISO documents $[18,19]$ being perhaps the most prominent examples. These documents recognize the influence of contact slide-roll ratio (SRR) on micropitting and attempt to account for this influence through the introduction of a 'sliding factor', $\mathrm{S}_{\mathrm{GF}, \mathrm{Y}}$ which is used to reduce the predicted EHL film thickness values as SRR increases. The proposed reasoning behind this approach is that the increased slide-roll ratio causes a drop in EHL oil film thickness, due to an increase of incontact flash temperature with increasing SRR, which in turn causes more micropitting. This theory is described in more detail in $[20,21]$. Although micropitting may well increase with increasing SRR, the relevant mechanism proposed in these documents contradicts some widely accepted aspects of elastohydrodynamic lubrication. In particular, it is far from clear why the in-contact flash temperature would have a significant effect on the EHL film thickness, given that it is well-established that EHL oil film thickness is a function of the lubricant viscosity in the inlet of the contact and therefore depends on the inlet contact temperature (see [22, 23] for example). The question that needs answering is therefore that of whether correlation means causation: in practice, correlation may indeed exist between increased level of sliding and increased amount of micropitting damage but the mechanism responsible for any correlation is not clear and warrants further scrutiny if appropriate design criteria are to be devised.

The above discussion serves to illustrate the need for better understanding of physical mechanisms responsible for micropitting, particularly in relation to the influence of slide-roll ratio. Consequently, the objective of this paper is to provide further insight into the influence of the magnitude and direction of slide-roll ratio on micropitting, with particular focus on the mechanisms at play. This is done by conducting appropriately designed experiments, where the influence of a single contact parameter of interest is isolated, together with numerical contact analyses to further elucidate the stress history experienced by the roughness asperities of the contacting surfaces at different slide-roll ratios. The findings described here can help in devising appropriate design guidelines to prevent micropitting failures in geared transmissions and other mechanical systems. 


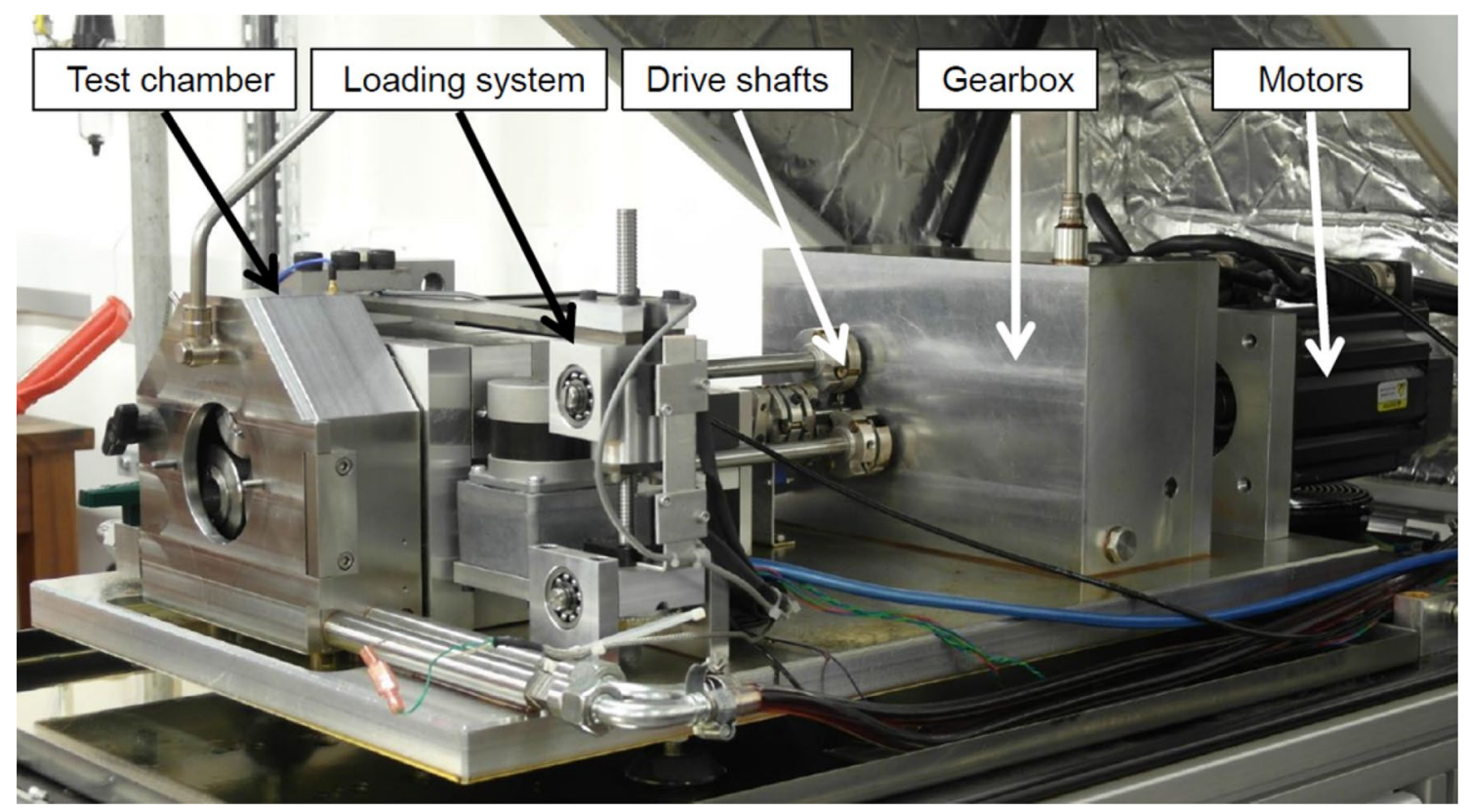

Fig. 1 General layout of the PCS instruments micropitting test rig (MPR)

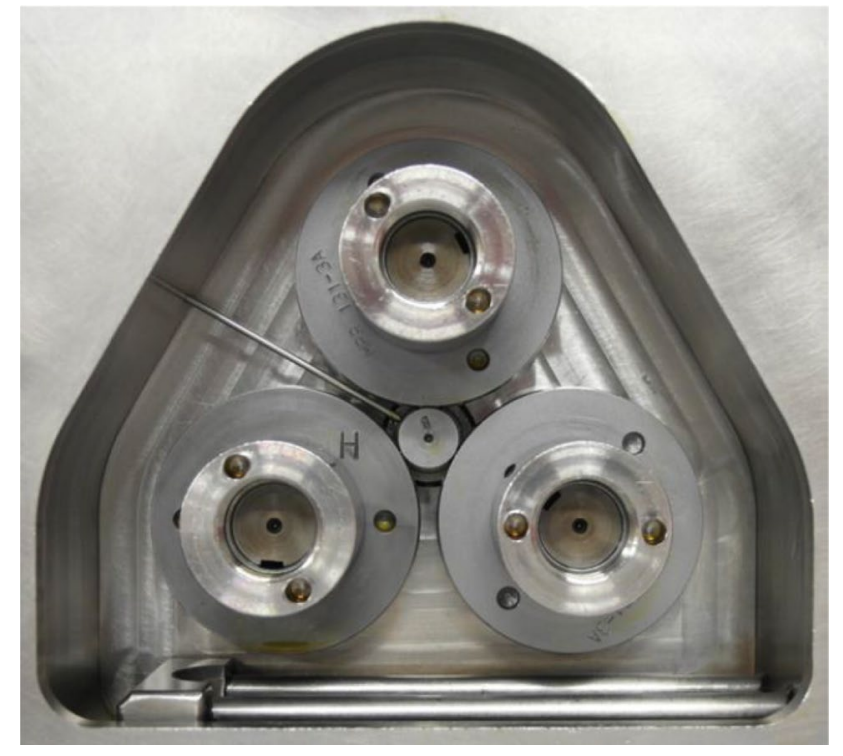

Fig. 2 Inside of the micropitting rig test chamber showing the central test roller and the three counterface discs

\section{Experimental Methods and Materials}

\subsection{The Micropitting Rig (MPR)}

Micropitting tests were conducted using a PCS Instruments Micropitting Rig illustrated in Fig. 1. The rig uses a triple contact design whereby a central roller specimen

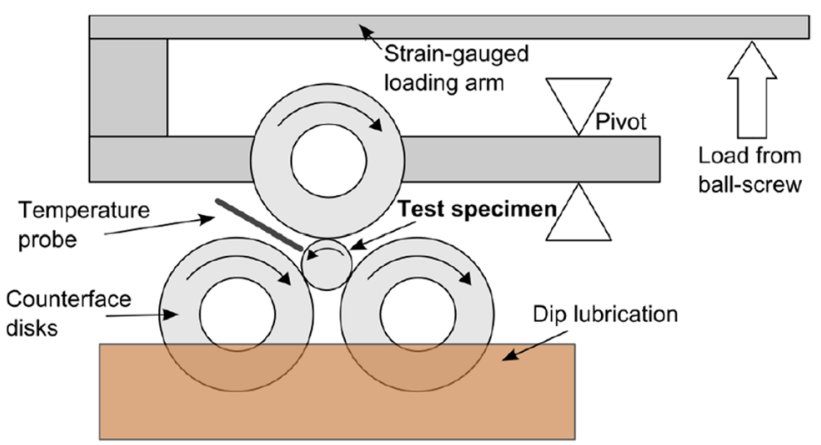

Fig. 3 A schematic of the micropitting test rig including the loading mechanism

is in contact with three counterface discs, resulting in three roller contact cycles per roller revolution. The specimen arrangement inside the test cell is shown in the photograph in Fig. 2, while the schematic diagram in Fig. 3 illustrates the loading and lubrication mechanisms employed in the rig. Load is applied to the roller specimen through the top disc using a motorized loading arm. The three counterface discs are located $120^{\circ}$ apart which results in equal reaction force at each of the three roller-disc contacts. The roller and counterface discs are driven by separate motors so that any slide-roll ratio in the range -200 to $+200 \%$ can be achieved. Lubrication is achieved using a temperature-controlled dip lubrication system, with the bottom two counterface discs partially submerged and dragging oil into all three contacts during operation. Oil sump temperature is measured using 


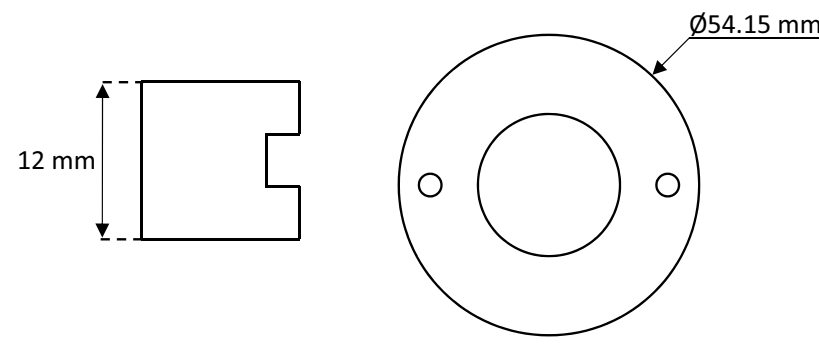

Fig. 4 Schematic diagrams of the micropitting test specimens: cylindrical test roller (left) and a crowned counterface disc (right)

Table 1 Nominal composition of $16 \mathrm{MnCr} 5$ steel used for present test specimens

\begin{tabular}{lllll}
\hline & $\mathrm{C}$ & $\mathrm{Si}$ & $\mathrm{Mn}$ & $\mathrm{Cr}$ \\
\hline Composition \% & $0.14-0.19$ & $<0.4$ & $1.1-1.3$ & $0.8-1.1$ \\
\hline
\end{tabular}

a resistance thermometer positioned within the test cell. The roller specimen drive shaft is equipped with a torque meter, which provides a continuous measurement of torque throughout the tests, thus enabling the average contact friction coefficient to be monitored.

In its current form, this experimental set-up was first successfully used by Olver and co-workers [4] although its origins can be traced to earlier studies by Graham [24]. The set-up has since been repeatedly proven to be suitable for studies of pitting and micropitting $[5,15,25]$.

\subsection{Test Specimens}

The specimens used in this study were a 12-mm-diameter cylindrical test roller and crowned counterface discs, where the crown radius transverse to rolling direction was $80 \mathrm{~mm}$ and the disc diameter in the rolling direction was $54.15 \mathrm{~mm}$. The specimens are illustrated in the schematic drawing of Fig. 4. This specimen configuration results in an elliptical contact geometry. This is in contrast to the 'standard' line contact geometry commonly employed on the PCS MPR rig which is formed with a 'chamfered' cylindrical roller and cylindrical counterface discs. The implications of the choice of MPR contact geometry are discussed in some detail by the present authors in [26]. For the purposes of this study, it should be noted that the employed elliptical contact geometry avoids edge stresses that are present in the 'standard' MPR line contact set-up, while also allowing the amount of micropitting wear to be measured more easily. On the other hand, the elliptical contact does mean that the initial contact pressure can reduce slightly over the course of the test if significant amount of wear is generated. All specimens were made of case-carburized $16 \mathrm{MnCr} 5$ gear steel with nominal composition shown in Table 1. The test roller case depth was approximately $0.9 \mathrm{~mm}$. The roller and disc were hardened to approximately $680 \mathrm{HV}$ and $750 \mathrm{HV}$, respectively. This hardness differential was previously shown to be suitable for controlled micropitting tests [27], since it ensures that the surface of the counterface discs remains largely unchanged over the test duration and the fatigue damage is preferentially generated on the test roller.

The surfaces of both the test rollers and counterface discs were circumferentially ground, that is the roughness lay was oriented in the rolling direction. Given the known influence of surface roughness on micropitting, the finish of the specimens was carefully controlled to minimize the variation between tests as much as possible. All surface roughness values were measured using a Taylor Hobson Talysurf stylus profilometer. The quoted roughness values are an average of four measurements for each specimen taken in the direction transverse to rolling direction. The as-received rollers and discs had an average nominal root mean square roughness, $R_{\mathrm{q}}=0.20 \pm 0.04 \mu \mathrm{m}$ and $R_{\mathrm{q}}=0.42 \pm 0.006 \mu \mathrm{m}$, respectively. It should be noted that the variation of the roller roughness is largely irrelevant since the roller is softer than the discs and its initial surface topography is very quickly modified through running-in and micropitting. In contrast, the roughness of the harder counterface discs is of major significance, since it persists during the test, and any significant roughness variations from specimen to specimen can affect the relative rates of micropitting.

\subsection{Test Lubricant}

All tests were performed with a custom-blended oil which consisted of polyalphaolephin (PAO) base-oil with zinc dialkyldithiophosphate (ZDDP) anti-wear additive blended in at a treat rate of $0.1 \mathrm{wt} \%$ phosphorus. A custom-blended oil with a known formulation was used here in preference to a fully-formulated commercial oil in order to avoid any effects of unknown oil additive packages. ZDDP anti-wear additive was added to oil in order to minimize mild wear of the surfaces, so that the counterface roughness persists throughout the test, thus promoting micropitting. This competition between mild wear and micropitting is well-known to have a crucial effect on the amount of micropitting under given conditions $[5,15]$ and the presence of ZDDP ensures that the outcome of this competition is controlled in favour of micropitting in the present tests since it is micropitting that is of interest here. The viscosity and density of the test oil were measured using a Stabinger viscometer to be $25 \mathrm{cSt}$, $0.8108 \mathrm{~g} / \mathrm{cm}^{3}$ at $40{ }^{\circ} \mathrm{C}$ and $5 \mathrm{cSt}, 0.7732 \mathrm{~g} / \mathrm{cm}^{3}$ at $100{ }^{\circ} \mathrm{C}$, respectively. Viscosities at other temperatures were then calculated using the ASTM D341-722 standard equation. It should be noted that the combined choice of oil, surface roughness and temperatures in the present tests was made 
to ensure that the specific film thickness (as defined below) was sufficiently low to cause micropitting so that the phenomenon could be studied.

\subsection{Micropitting Test Procedure}

Test specimens and fasteners were ultrasonically cleaned first in toluene and then isopropanol for $15 \mathrm{~min}$ prior to testing. Before each test the test chamber of the rig was cleaned using toluene followed by isopropanol and then dried. In order to remove all traces of lubricant from previous

Table 2 Roller inspection intervals in the current micropitting test procedure

\begin{tabular}{lll}
\hline $\begin{array}{l}\text { Test step } \\
\text { number }\end{array}$ & $\begin{array}{l}\text { Number of roller contact } \\
\text { cycles } \times 10^{6}\end{array}$ & $\begin{array}{l}\text { Cumulative number of } \\
\text { roller contact cycles } \times 10^{6}\end{array}$ \\
\hline 1 & 0.09 & 0.09 \\
2 & 0.42 & 0.51 \\
3 & 0.42 & 0.93 \\
4 & 0.84 & 1.77 \\
5 & 0.84 & 2.60 \\
6 & 0.84 & 3.44 \\
7 & 0.84 & 4.28 \\
8 & 0.84 & 5.12 \\
\hline
\end{tabular}

experiments, a flushing step was performed with the test lubricant prior to running the actual tests.

All tests were conducted to the same number of 5.12 million contact cycles seen by the test roller specimen. In order to make a valid comparison of micropitting damage at different slide-roll ratios (and hence different roller rotational speeds), the number of load cycles for the roller is calculated using the actual roller speed in each case. In addition, the test durations were adjusted as needed so that the total number of roller contact cycles was the same (5.12 million) for all slide-roll ratios used. The tests were interrupted at set intervals, specified in Table 2, in order to measure surface profiles and take optical micrographs of the test roller surface. At each inspection interval, four surface profile measurements of the roller specimen were taken across the running track (transverse to rolling direction) using a Taylor Hobson Talysurf stylus profilometer. The measurements were used to quantify the amount of micropitting wear at each step. Figure 5 shows a typical wear profile and illustrates how the value of maximum micropitting wear depth is obtained from it. In addition, surface profiles of counterface discs were also measured during selected inspection intervals in order to confirm that no wear or excessive reduction in roughness of the discs has occurred due to mild wear. This was confirmed in all cases.
Fig. 5 An example surface profile of a micropitted roller test specimen measured in the direction transverse to rolling. The maximum micropitting wear depth indicated on the figure was used as a parameter describing micropitting severity at each inspection step

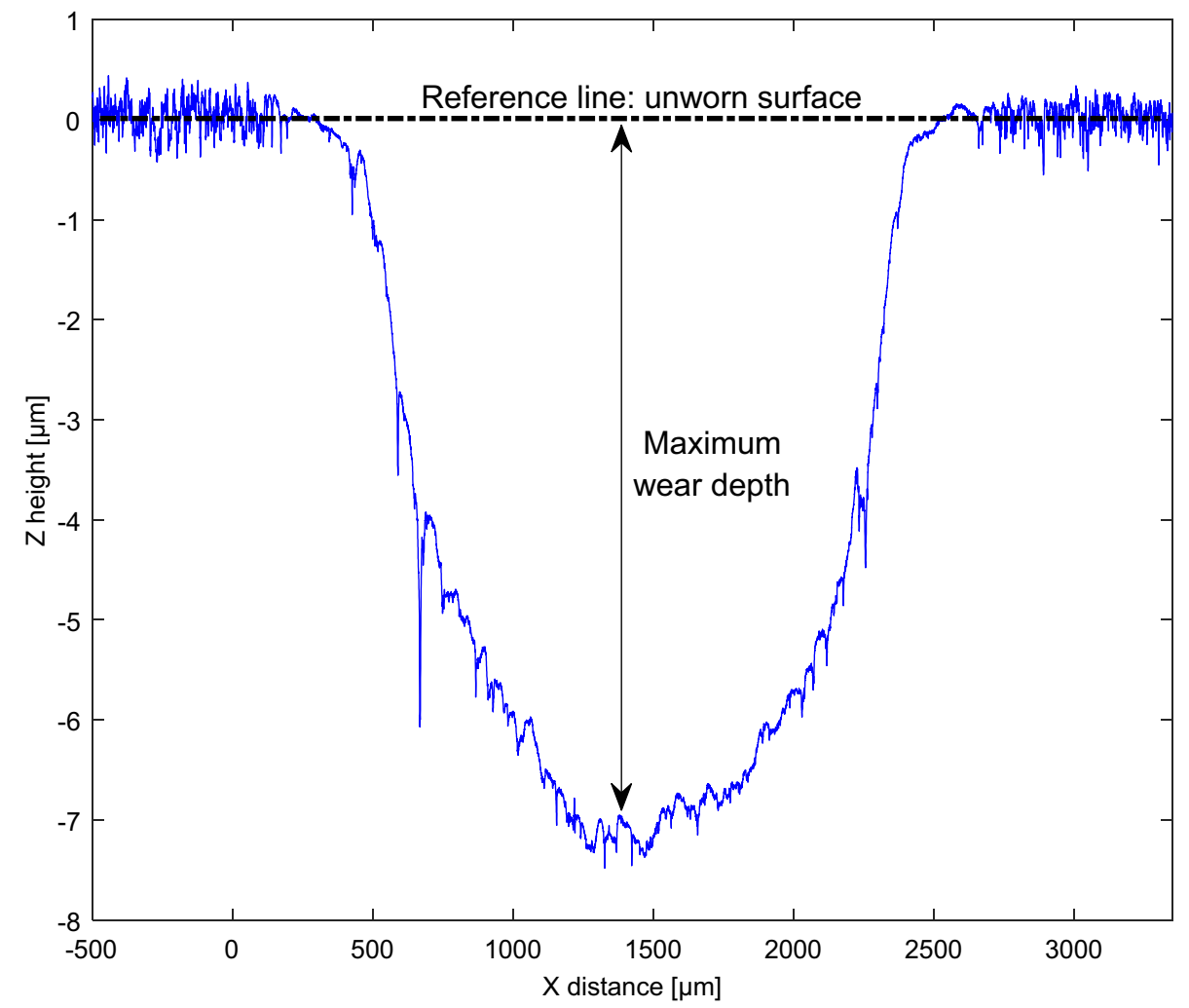




\subsection{Isolating the Influence of Slide-Roll Ratio on Micropitting by Accounting for Thermal Effects}

Given that the primary aim of this study was to evaluate potential effects of the slide-roll ratio (SRR) on the severity of micropitting, the test procedure was designed to closely control all other test parameters while only varying the slide-roll ratio. In particular, the increase of specimen bulk temperature associated with higher frictional heat input at increased SRRs had to be accounted for. This is important because a rise in bulk temperature produces an increase of contact inlet temperature and consequently reduces the oil viscosity at the contact inlet, leading to a reduced EHL film thickness. The same sequence of events occurs in real machine elements, particularly in gears where the SRRs are relatively high. The importance of this thermal response in gear testing is discussed in detail by Olver [28]. Given the strong relationship between the lubricant film thickness and micropitting, it is clear that if the influence of SRR alone on micropitting is to be isolated and understood, the test procedure must be designed to keep the film thickness, and hence the lambda ratio, the same for all tested SRRs. This is done in the present tests by calculating the bulk temperature increase of the specimens at each SRR and then adjusting the oil sump temperature accordingly so that the contact inlet temperature is kept the same regardless of SRR. The method is outlined below.

Accurate prediction of temperatures in lubricated rolling-sliding EHL contacts is not straight forward. CFD methods can be used to obtain complete temperature distribution [29] but given the computational complexities involved, this approach is only really feasible for single contacts and not practical in the case of real components, particularly when bulk temperatures are of interest. Given these difficulties, various approximations are commonly used to provide rough estimates in affected applications, notably in gears [30]. In this case, the difficulties arise mainly owing to the fact that the coefficient of friction is usually not known, and that it is very difficult to calculate heat transfer coefficients for gears rotating in a two phase mixture of air and oil. However, it is possible to provide relatively accurate estimates of bulk temperature increase if friction is known, i.e. measured, and the geometry of the contacting components is such that heat transfer coefficients can be estimated from basic heat transfer relationships. A suitable method was outlined by Olver [28] and extended by Olver and Spikes [31] who applied it to a contact of two discs. Kadiric et al. [32] used a similar approach to predict tooth bulk temperatures for a pair of meshing spur gears. Here the approach is adapted to predict bulk temperature increase of the discs and the roller in the present triple-disc test rig.

The slide-roll ratio (SRR) is defined here as
$\mathrm{SRR}=\frac{\text { sliding speed, } \Delta v}{\text { entrainment speed, } v_{\mathrm{e}}}=\frac{v_{1}-v_{2}}{\left(v_{1}+v_{2}\right) / 2}$,

where $\mathrm{v}_{1}$ and $\mathrm{v}_{2}$ are the surface speeds of the roller and disc, respectively. Since the direction of sliding is of significance in micropitting, the reader should note that the SRR definition in Eq. 1 is such that a negative value of the slide-roll ratio means that the roller is slower than the disc.

The rate of frictional heat generated in a rolling-sliding contact between the roller and the disc is given by

$q^{\prime}=\mu W|\Delta v|$,

where $q^{\prime}$ [Watts] is the rate of heat generated, $\mu$ is the friction coefficient, $W[\mathrm{~N}]$ is the normal load and $\Delta v[\mathrm{~m} / \mathrm{s}]$ is the sliding speed. Clearly, increasing the SRR at a constant entrainment speed results in a larger amount of heat generation since the sliding speed, $\Delta v$, is increased.

Figure 6 illustrates the various temperatures of relevance to EHL contacts, namely mean in-contact oil film temperature, $T_{\text {oil }}$, mean in-contact surface flash temperatures, $T_{\mathrm{f} 1}$ and $T_{\mathrm{f} 2}$, bulk temperatures of the two surfaces, $T_{\mathrm{b} 1}$ and $T_{\mathrm{b} 2}$ (which may be different if the two surfaces have different speeds), and the inlet temperature (mean bulk temperature), $T_{\mathrm{b}}$. The situation presented is analogous to a gear tooth contact where $T_{\mathrm{b}}$ is the mean bulk tooth temperature and is often estimated by either assuming that it is $20^{\circ} \mathrm{C}$ higher than the sump temperature $\left(T_{\mathrm{A}}\right)$ or by using approximate relationships such as that in [30] which estimates that $T_{\mathrm{b}}=T_{\mathrm{A}}+0.56 \Delta T_{\mathrm{f}, \max }$, where $\Delta T_{\mathrm{f}, \max }$ is the maximum flash temperature rise along the path of contact during one mesh

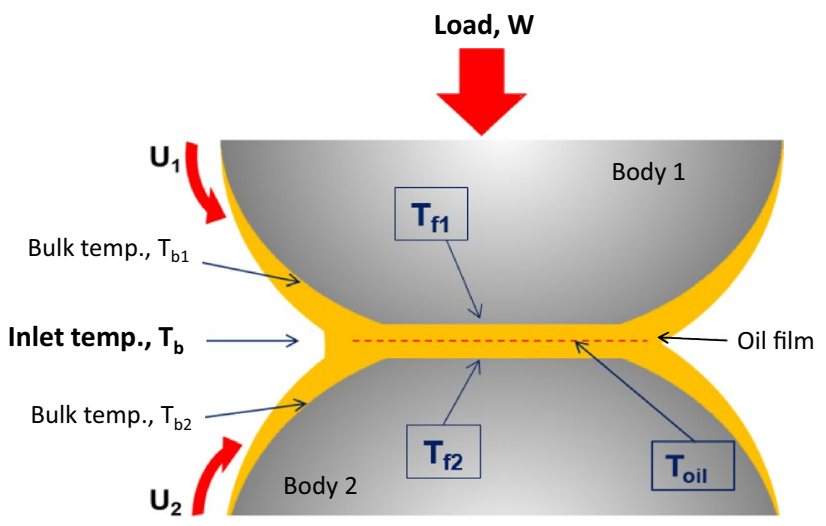

Fig. 6 Contact temperatures in an elastohydrodynamic (EHL) contact: mean oil film temperature, $T_{\text {oil }}$, mean in-contact surface flash temperatures, $T_{\mathrm{f} 1}$ and $T_{\mathrm{f} 2}$, bulk temperatures of the two surfaces, $T_{\mathrm{b} 1}$ and $T_{\mathrm{b} 2}$, and the mean bulk (inlet) temperature, $T_{\mathrm{b}}$, which is used for the film thickness predictions. $U_{1}$ and $U_{2}$ are the surface speeds of the two contacting surfaces, the roller and the disc in the case of the MPR rig or two meshing gear teeth for example 
cycle. The method presented here attempts to provide more accurate predictions applied to the MPR rig.

The frictional heat generated in the contact between the roller and the disc specimens is conducted away into their surfaces (the heat convection by oil is negligible particularly at low specific film thicknesses). As a result, the bulk surface temperature $T_{\mathrm{b}}$ rises above the oil sump temperature $T_{\mathrm{a}}$. The bulk temperature of the roller $\left(T_{\mathrm{b} 1}\right)$ and a disc $\left(T_{\mathrm{b} 2}\right)$ are, respectively,

$T_{\mathrm{b} 1}=T_{\mathrm{a}}+3 \alpha q^{\prime} M_{1}$

$T_{\mathrm{b} 2}=T_{\mathrm{a}}+(1-\alpha) q^{\prime} M_{2}$,

where $\alpha$ is the proportion of heat conducted into the roller in each of the three contacts (hence, the proportion $(1-\alpha)$ is conducted into each of the counterface discs) and $M_{1}$ and $M_{2}$ are the steady-state thermal resistances of the roller and a disc, respectively. $M_{1}$ and $M_{2}$ depend on the geometry and the heat transfer coefficients of the roller and discs. The latter is difficult to calculate accurately but can be estimated using known heat transfer expressions for discs as described by Olver [28].

The value of the heat partition coefficient, $\alpha$, is found by equating the mean contact temperature of the two bodies as first suggested by Jaeger [33]. Under conditions where film thickness is low enough for significant solid-to-solid contact to occur, as is the case here, the temperature difference across the oil film can be ignored (i.e. $\Delta T_{\text {oil }}=0$ ) so that the mean in-contact temperatures of the two bodies can be assumed to be the same, i.e.

$T_{\mathrm{b} 1}+\Delta T_{\mathrm{f} 1}=T_{\mathrm{b} 2}+\Delta T_{\mathrm{f} 2}=T_{\mathrm{c}}$.

where $T_{c}$ is the mean contact temperature. This approach of assuming the mean contact temperature of the two bodies to be equal is a widely accepted one. However, in reality the two bodies each have a distribution of contact temperatures so that a full treatment requires that the temperatures are matched at each local point which in turns affects the heat partition fraction which in itself varies locally [34]. Given the numerous factors that affect the heat flows in the MPR test rig and the relatively small SRR employed, the use of the accepted assumption of equal mean temperatures is justified here.

The heat flow described here is represented by the thermal network shown in Fig. 7.

The mean flash temperature rise, $\Delta T_{f}$, of the roller and discs can be calculated using expressions provided by Jaeger [35]:

$\Delta T_{f, i}=\frac{1.06 a_{i} q^{\prime}}{A k_{i}}\left(\frac{\chi_{i} b}{v_{i}}\right)^{1 / 2}$,

where subscript $i$ is either 1 or 2 identifying the roller or the disc, $A$ is the contact area, $b$ is the contact semi-width in rolling direction, $k$ is the heat conductivity of the material and $\chi$ is the diffusivity of the material (i.e. $\chi=k / \rho c$, where $\rho$ and $c$ are the density and specific heat capacity).

Substituting the expressions in Eqs. 3, 4 and 6 into Eq. 5 and, for the sake of simplicity, denoting the transient thermal resistances of the roller and the disc as $B_{i}=\frac{1}{A k_{i}}\left(\frac{\chi_{i} b}{v_{i}}\right)^{1 / 2}$, the heat partition fraction, $\alpha$, can now be found as

$\alpha=\frac{1.06 B_{2}+M_{2}}{1.06\left(B_{1}+B_{2}\right)+\left(3 M_{1}+M_{2}\right)}$.

This then allows the bulk temperatures of the roller and the disc, $T_{\mathrm{b} 1}$ and $T_{\mathrm{b} 2}$, to be found. Since the roller and discs have different speeds (as determined by the imposed slide-roll ratio), the mean oil temperature in the inlet, $T_{\mathrm{b}}$,

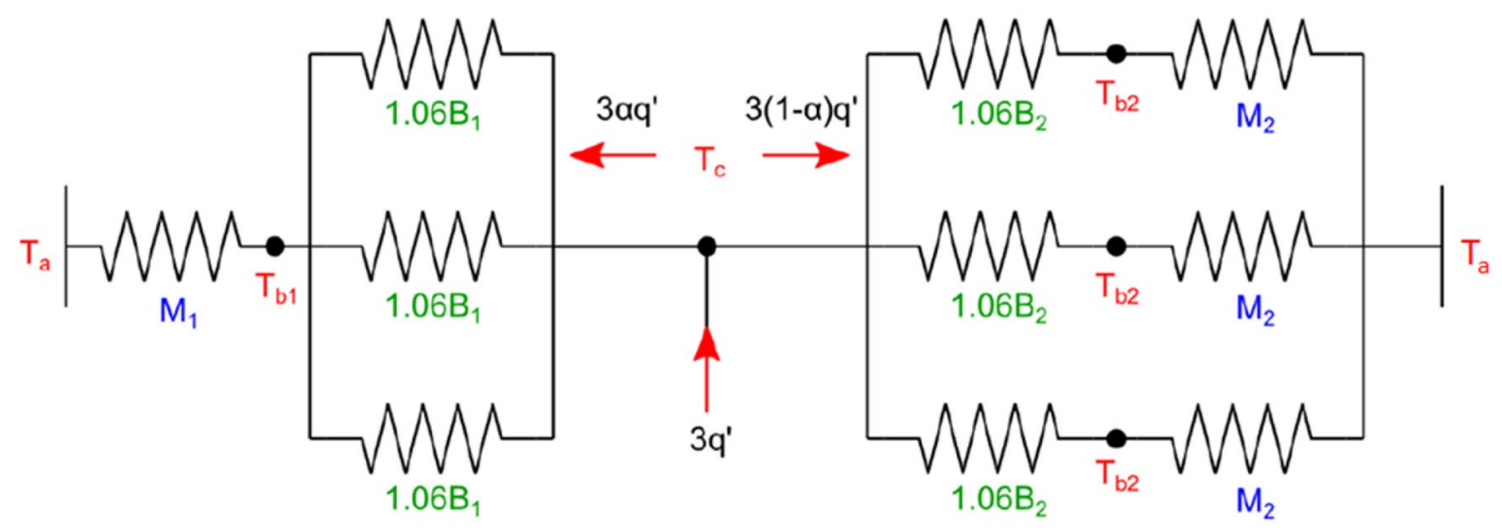

Fig. 7 Thermal network for the three ring-roller contacts in the MPR rig. $M_{1}$ and $M_{2}$ are the steady-state thermal resistances of the roller and a disc, respectively, $B_{1}$ and $B_{2}$ are the transient thermal resistances of the roller and a disc, respectively, $q^{\prime}$ is the frictional heat flux generated in each of the three ring-roller contacts, and $\alpha$ is the heat partition fraction. $T_{\mathrm{a}}, T_{\mathrm{b}}$ and $T_{\mathrm{c}}$ are ambient (sump), bulk and mean contact temperatures, respectively 
is found as a speed weighted average of the two bulk temperatures, i.e.

$T_{\mathrm{b}}=\frac{v_{1} T_{\mathrm{b} 1}+v_{2} T_{\mathrm{b} 2}}{v_{1}+v_{2}}$

In the present study, the above analysis is applied to each set of test conditions used and the value of oil sump temperature, $T_{\mathrm{a}}$, adjusted in each case so that the oil inlet temperature, $T_{\mathrm{b}}$, and therefore the film thickness, is the same in all tests regardless of the test conditions imposed. This allows for any potential thermal effects to be eliminated so that the influence of slide-roll ratio can be studied in isolation.

\subsection{Test Conditions}

Test conditions were chosen to be representative of steel gear applications as well as to ensure that micropitting damage was produced in preference to excessive mild wear. Occurrence of mild wear makes it difficult to study micropitting as they are competing damage mechanisms: excessive mild wear, particularly of the harder counterface, inhibits crack initiation and propagation $[5,15]$. The outcome of the competition between mild wear and micropitting is heavily influenced by the specific film thickness ( $\Lambda$ ratio). Previous research has shown that micropitting can reliably be generated in the micropitting rig at $\Lambda$ ratios of about 0.12 and less $[5,15]$ and relatively low contact pressures. Consequently, the present study was conducted with $\Lambda$ ratio of 0.1 in all cases and maximum Hertzian contact pressure of $1.78 \mathrm{GPa}$ and 1.4 GPa, both of which are representative of typical gear teeth contacts. The predicted value of the minimum EHL film thickness in all tests was $43 \mathrm{~nm}$ using Dowson and Hamrock EHL film thickness equation [36] with the oil pressure-viscosity coefficient of $15 \mathrm{GPa}^{-1}$ and the inlet oil temperature predicted as described above. Slide-roll ratios of $15 \%, 5 \%,-5 \%,-15 \%,-30 \%$ were chosen. This range covers typical slide-roll ratios found in gear applications. The speeds of discs and the roller were set appropriately in each case to achieve the desired SRR but the entrainment speed itself was kept constant at $2 \mathrm{~m} / \mathrm{s}$ in all tests. Table 3 lists the complete set of conditions used in the present study. It is evident in the table that the sump oil temperature was adjusted between tests to account for the effects of bulk heating at different slide-roll ratios and loads so that the inlet oil temperature was the same $\left(106^{\circ} \mathrm{C}\right)$ in all cases to give the same $\Lambda$ ratio of 0.1 in all tests. The control of bulk heating effects was achieved following the procedure outlined earlier. Any additional effects of load changes on film thickness were also accounted for through sump temperature adjustment but, given the insensitivity of EHL film thickness to the applied load, these are minimal and not apparent in the quoted inlet temperature values rounded to the nearest whole number. Finally, measured average friction coefficient was about 0.09 in all tests. The minimal variation in friction between tests was a direct result of keeping the $\Lambda$ ratio constant and is important since the level of friction is known to influence the extent of micropitting damage [16].

The definition of the $\Lambda$ ratio in this paper is based on the minimum film thickness and the counterface disc roughness only, rather than compound roughness of the disc and the roller specimen, i.e.

$\Lambda=\frac{h_{\mathrm{min}}}{R_{\mathrm{q}, \mathrm{disc}}}$,

where $h_{\min }[\mathrm{m}]$ is the minimum oil film thickness and $R_{\mathrm{q}}$ is the root mean square roughness [m]. Excluding the roughness of the test roller from the definition is more representative of the contact conditions throughout the duration of the test since the initial roughness of the roller is quickly modified due to damage so including its initial value is largely meaningless. In contrast, the roughness of the harder counterface persists throughout the test duration. This definition of $\Lambda$ ratio is also consistent with previous micropitting studies using the MPR rig $[4,5,16]$.
Table 3 Test conditions used in the current micropitting study

\begin{tabular}{|c|c|c|c|c|c|c|c|}
\hline Slide-roll ratio $2 \times\left(\mathrm{U}_{\text {roller }}-\mathrm{U}_{\text {disc }}\right) /\left(\mathrm{U}_{\text {roller }}+\mathrm{U}_{\text {disc }}\right)$ & -0.3 & -0.15 & -0.15 & -0.05 & -0.15 & 0.05 & 0.15 \\
\hline Entrainment speed $(\mathrm{m} / \mathrm{s})\left(\mathrm{U}_{\mathrm{disc}}+\mathrm{U}_{\text {roller }}\right) / 2$ & 2 & & & & & & \\
\hline Load $(\mathrm{N})$ & 500 & 500 & 500 & 500 & 240 & 500 & 500 \\
\hline Max Hertz contact pressure (GPa) & 1.78 & 1.78 & 1.78 & 1.78 & 1.40 & 1.78 & 1.78 \\
\hline Sump temperature $\left({ }^{\circ} \mathrm{C}\right)$ & 77 & 89 & 89 & 100 & 94 & 100 & 85 \\
\hline $\begin{array}{l}\text { Contact inlet temperature (calculated using the } \\
\text { method outlined in Sect. } 2.5)\left({ }^{\circ} \mathrm{C}\right)\end{array}$ & 106 & & & & & & \\
\hline $\begin{array}{l}\text { Measured coefficient of friction (quoted value is } \\
\text { the average over test duration) }\end{array}$ & $\sim 0.09$ & & & & & & \\
\hline Predicted minimum film thickness, $h_{\min }(\mathrm{nm})$ & 43 & & & & & & \\
\hline Lambda ratio $\left(=h_{\min } / R_{\mathrm{q}, \text { disc }}\right)$ & 0.1 & & & & & & \\
\hline
\end{tabular}




\subsection{Lubricant Film Thickness Measurements}

An optical EHD test rig (PCS Instruments EHD 2 rig) was used to investigate the effect of slide-roll ratio on elastohydrodynamic film thickness with the same model oil that was used in micropitting tests. These film thickness results were needed when assessing the merits of potential mechanisms that may be responsible for the observed increase in micropitting with increasing SRR, as will be discussed later. The method utilizes an optical interferometry technique to measure the oil film thickness between a steel ball (or a barrel) and a glass disc in a sliding-rolling contact. The ball and disc are held in a temperature-controlled lubricant bath. In the present study it was desired to obtain a complete map of lubricant film in the contact in order to study both the central and minimum film thickness values, so the optical interferometry rig was operated with the spacer layer imaging method (SLIM) [37]. The standard configuration of this rig employs a ball-on-disc set-up. However, the present study employed an additional configuration of a barrel roller on glass disc which results in an elongated elliptical contact and therefore closely mimics the elliptical contact geometry used in the MPR micropitting tests, thus enabling the two sets of results to be directly related.

\section{Results}

\subsection{Observed Morphology of Micropitting Damage}

Micropitting damage was successfully produced on the test rollers at all conditions employed here. Examination of the roller surface throughout the test (as described in Table 2) with stylus profilometer and an optical microscope showed that the roller radius was progressively reduced due to micropitting damage on the surface. At the end of the test, the roller surfaces were also inspected using a scanning electron microscope (SEM). Figure 8 shows SEM micrographs of roller specimen surfaces tested at different SRRs. In all cases, the surfaces contained a high density of uniformly spaced V-shaped cracks and small pits. The cracks were oriented transversely to the rolling direction and their size was up to $50 \mu \mathrm{m}$. In some cases adjacent cracks coalesce or overlap each other. This damage morphology was consistent along the whole circumference of the specimens and covered the entire width of the running track.

Metallographic sections of test rollers were prepared by cutting through the centre of the running tracks along the direction of rolling, followed by polishing. Figure 9 shows example SEM micrographs of such roller sections for $\mathrm{SRR}=-0.05$ and +0.05 , where typical crack growth into the subsurface is clearly visible, while Fig. 10 shows examples of typical micropits apparent on these sections. It is evident that cracks generally form a shallow angle with

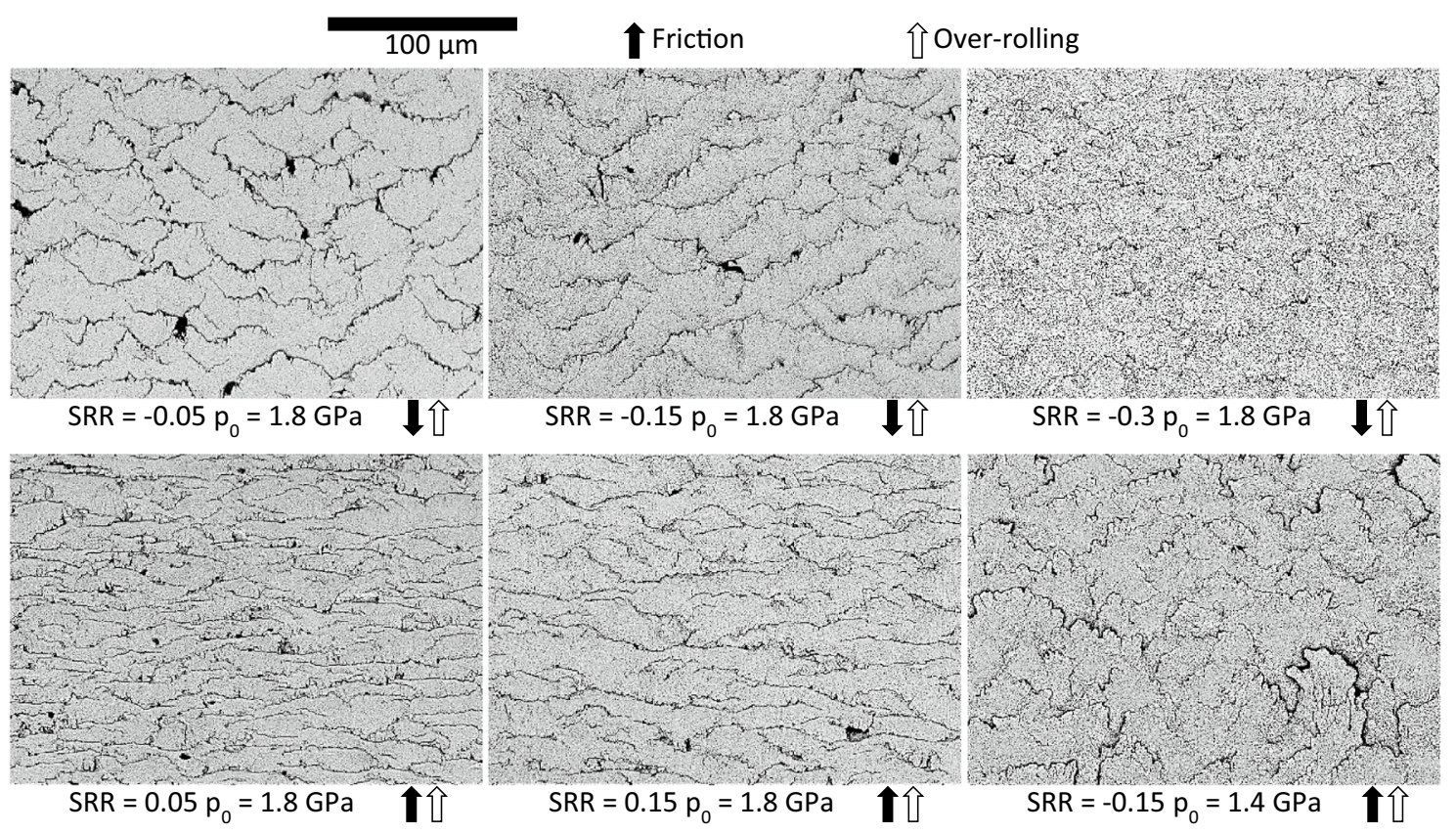

Fig. 8 SEM images of typical micropitted roller surfaces from the current tests, showing shallow micropits and numerous V-shaped cracks growing in opposite direction to applied friction force 


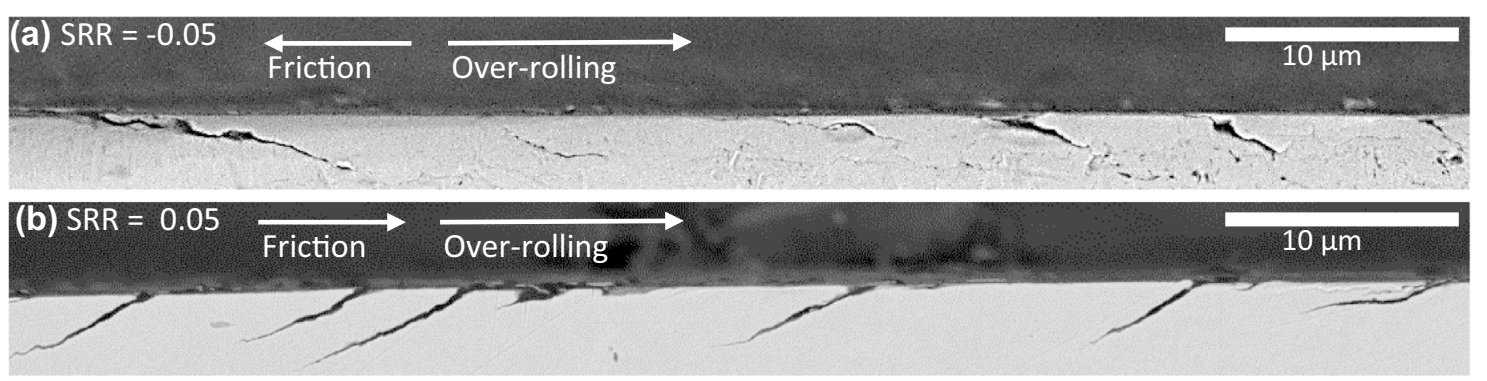

Fig. 9 An SEM image of a cross section (in rolling direction) of typical examples of micropitted roller specimens showing surface-breaking cracks growing at a shallow angle into the surface: $\mathbf{a} S R R=-0.05$ test and $\mathbf{b} S R R=+0.05$ test
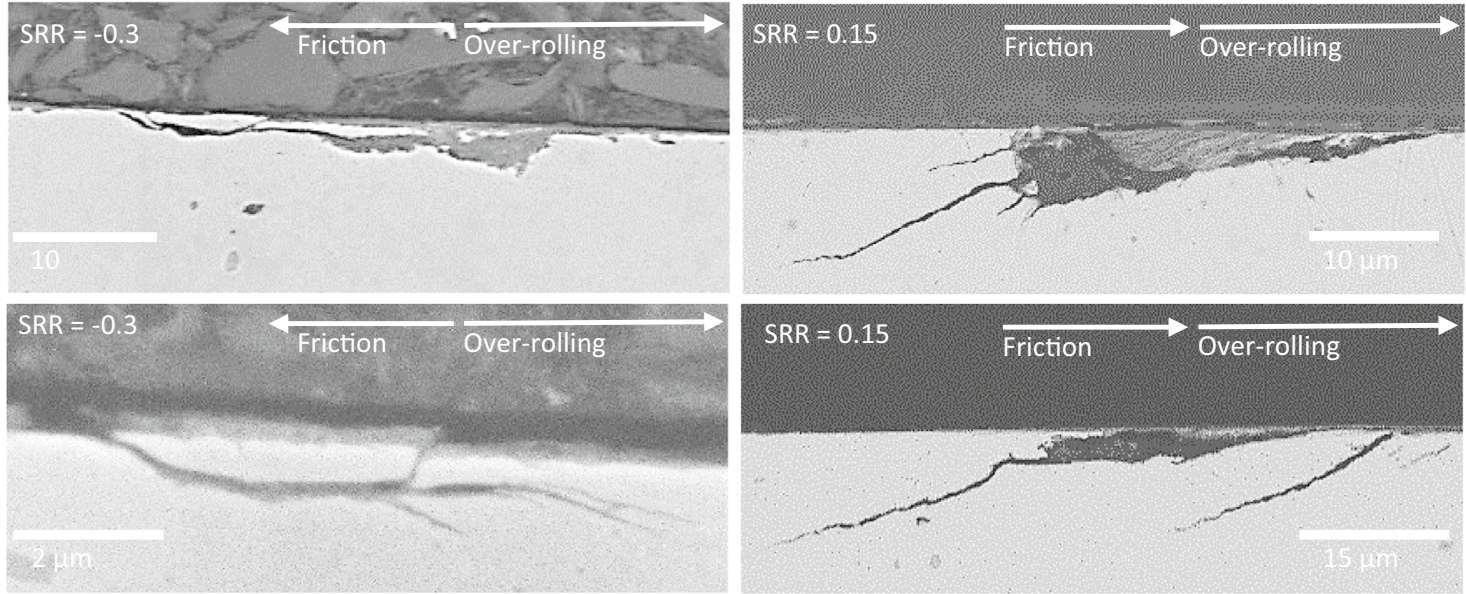

Fig. 10 SEM images of test rollers sectioned in rolling direction showing typical morphology of micropits

the surface and have a length of approximately 10-20 $\mu \mathrm{m}$. The direction of friction and contact motion is also indicated in Figs. 8, 9 and 10. It is immediately apparent that cracks grow in the direction opposite to the direction of the applied friction force. Cracks initially extend into the material eventually forming a micropit with a depth of approximately $5-10 \mu \mathrm{m}$ as seen in Fig. 10. The general appearance of micropitting damage described here was very similar across all SRRs and pressures employed in this study. These characteristics of micropitting cracks are in line with general appearance of micropitting on gear teeth surfaces and have been observed previously for surface-initiated rolling contact fatigue cracks $[1,15,25,38]$.

\subsection{Influence of SRR on Progression of Micropitting}

The progression of test roller diameter loss due to micropitting damage was recorded in all tests listed in Table 3. The progression of micropitting was quantified by measuring surface profiles across the running track on roller specimens at specified intervals throughout the test as outlined in Table 2. As an example, Fig. 11 shows a series of surface

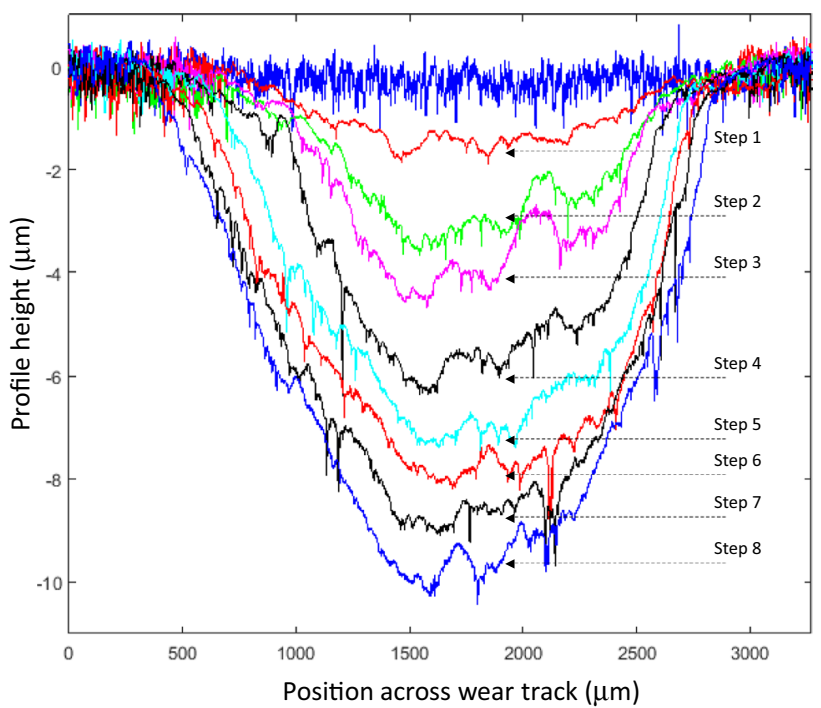

Fig. 11 Example of a typical set of test roller surface measurements taken during the test, showing the progression of micropitting wear at a slide-roll ratio of -0.15 (the number of cycles corresponding to each step are listed in Table 2) 
measurements for the test performed at $S R R=-0.15$, illustrating the progression of micropitting wear with increasing number of test cycles. Measurements at other SRR levels show similar behaviour in terms of continuous loss of profile but with different absolute values of micropitting wear depth at equivalent test stages.

This approach of quantifying the progression of micropitting damage is in line with that employed by Olver and coworkers $[4,5]$ on the same experimental set-up. It is worth noting here that in practical situations it is such progression of micropitting damage that leads to component failure, for example a significant loss of a gear tooth profile my lead to eventual gear failure. The alternative approach of generating only a few micropits locally on the surface of the test specimen and subsequently expressing the extent of micropitting damage as the proportion of studied area occupied by micropits, rather than the actual loss of material, is less applicable to practical situations since existence of a limited number of isolated micropits on the surface of a component such as a gear is generally not detrimental to its integrity; this alternative approach is also somewhat subjective since it is strongly dependent on the exact choice of the surface area over which micropitting is to be assessed.

The described profile measurements were taken at four different positions around the roller circumference for each test roller at each test interval. The parameter used to quantify the severity of micropitting was the average maximum depth of the wear track relative to the unworn, out of contact portion of the specimen as shown in Fig. 5. This wear depth was then plotted against the number of roller load cycles for each case. Figure 12 presents a plot of this average maximum micropitting wear depth for tests at $S R R=-0.05$, -0.15 and -0.3 all at Hertz pressure of $1.78 \mathrm{GPa}$. The results of two repeats for $S R R=-0.15$ are shown indicating good repeatability. It can clearly be seen in Fig. 12 that increasing SRR resulted in higher micropitting wear for a given number of loading cycles. The deepest final wear track was recorded in the test with $S R R=-0.3$. The likely reasons for the observed increase in micropitting wear with increasing SRR are considered in the discussion section.

To better understand these observations, the influence of sliding magnitude was analysed further. Since the entrainment speed was constant across all tests, increasing the SRR results in higher sliding speeds in the contact. Consequently, with increasing SRR the test specimens accumulated a longer sliding distance at equivalent numbers of contact cycles. Sliding distance is defined here as a product of sliding speed and test duration. Figure 13 plots the average maximum micropitting wear depth as a function of accumulated sliding distance. It can be seen that the micropitting wear curves at all SRR now collapse onto one master curve, i.e. the level of micropitting damage at a given sliding distance is the same for all SRRs. This

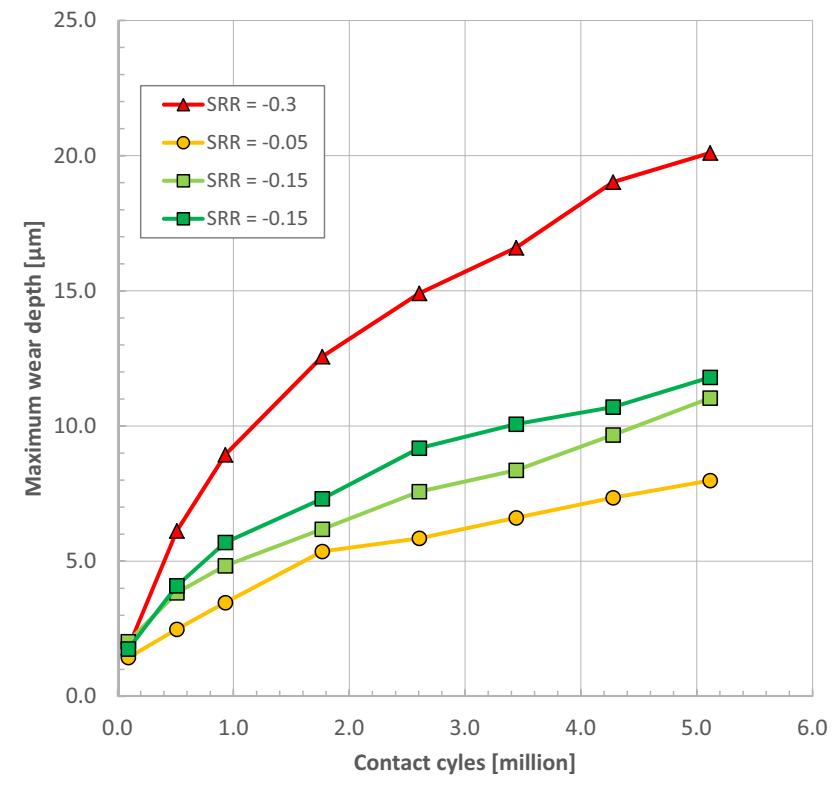

Fig. 12 Average maximum wear track depths measured at different levels of negative slide-roll ratio plotted against the number of roller macro-contact cycles; $p_{0}=1.78 \mathrm{GPa}$

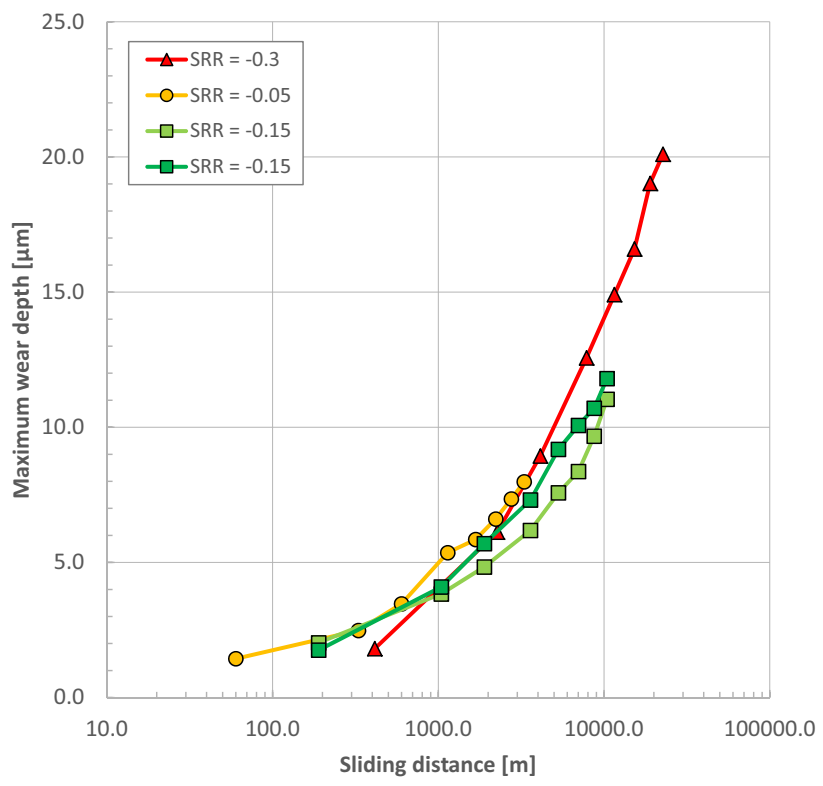

Fig. 13 Average maximum micropitting wear depths measured at different levels of negative slide-roll ratio plotted against the sliding distance (=sliding speed $\times$ test time); $p_{0}=1.78 \mathrm{GPa}$

observation clearly suggests that it is not the SRR itself that directly determines the level of damage but instead, the influence of SRR is via the increased sliding distance. Clearly, when all other variables are excluded, there exists a strong correlation between sliding distance and micropitting wear severity. The mechanisms responsible for this relationship are explained in the discussion. 
The measured evolution of friction coefficient for tests at different SRRs is shown in Fig. 14. It is evident that any apparent differences in friction between different tests are minimal, particularly considering the long test duration, with an average value of about 0.09 being representative of all tests. The small steps in friction traces apparent at specific times correspond to inspection intervals (specified in Table 2) when the roller was removed for damage inspection and subsequently placed back in the rig to continue the test. These are in part an artefact of the way the rig records friction but regardless, the overall long-term friction trend is unaffected. It should also be noted that, following the initial and rapid drop in friction due to wearing-in, friction remains relatively steady over the test duration which covers more than 5 million cycles.

\subsection{Influence of Sliding Direction on Progression of Micropitting}

The effect of direction of sliding velocity (i.e. positive or negative slide-roll ratio) on the micropitting damage was also studied. Figure 15 plots the progression of average maximum micropitting wear depth against roller load cycles for two levels of positive and negative SRR, namely \pm 0.05 and \pm 0.15 , with all other contact conditions being equal. Although micropitting was recorded for both sliding directions, it is immediately evident that at both absolute values of SRR significantly more micropitting wear was recorded for the negative value of SRR, i.e. when the roller is slower than the counterface ring. Reversing the direction of sliding reverses the direction of resultant traction force on the roller with respect to the direction of contact movement. For negative SRR, traction and contact movement direction are opposite to each other, while for positive SRR they are the same. Although we are here concerned with very short cracks leading to micropitting damage, this trend of negative

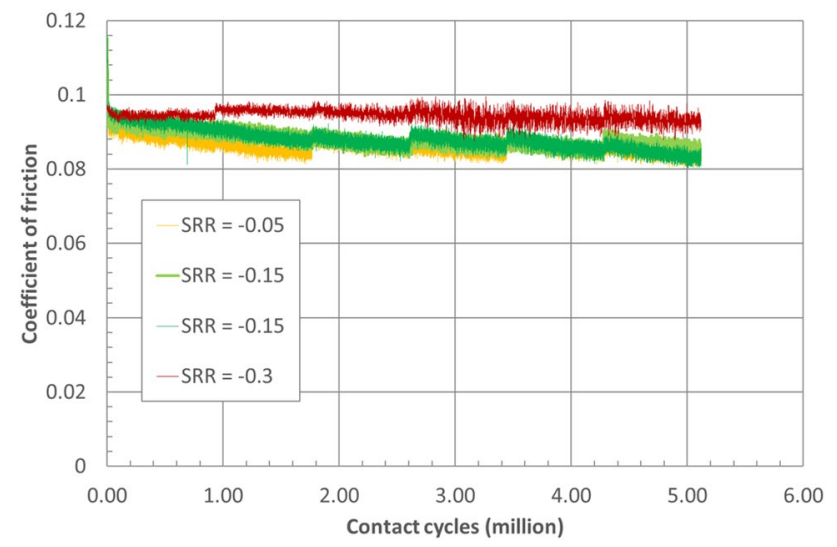

Fig. 14 Measured evolution of friction coefficient for tests at different SRRs; $p_{0}=1.78 \mathrm{GPa}$

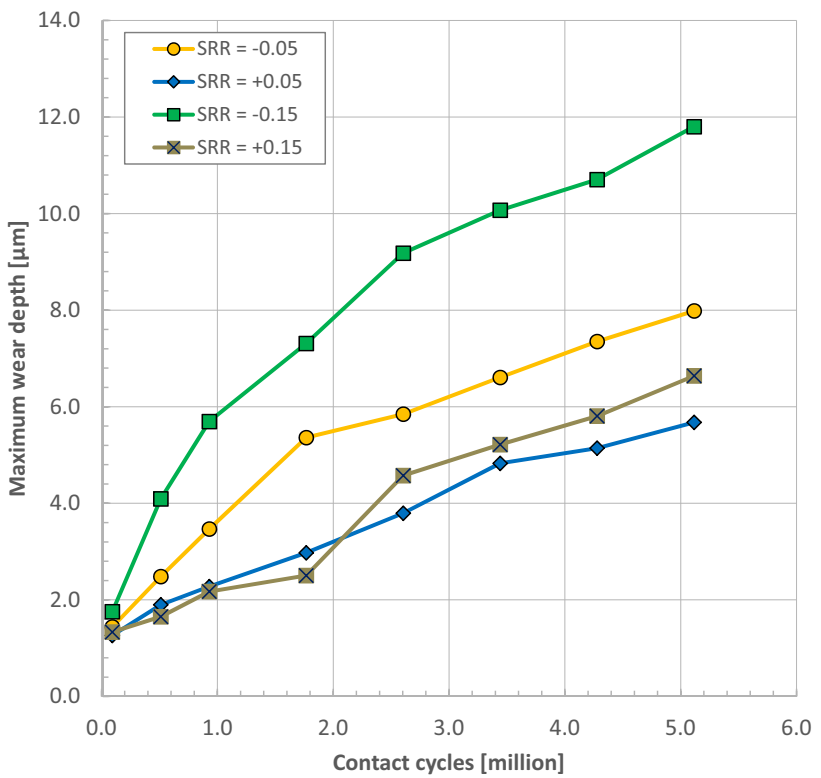

Fig. 15 Comparison of average maximum micropitting wear depths measured at positive and negative slide-roll ratios of \pm 0.05 and \pm 0.15 plotted against the number of roller macro-contact cycles; $p_{0}=1.78 \mathrm{GPa}$

sliding being relatively more damaging is also in line with previous observations of progression of surface-initiated pitting damage $[1,38,39]$. Figure 16 plots the maximum micropitting wear depth for the negative and positive SRR of 0.05 and 0.15 against the sliding distance in the same manner as done earlier in Fig. 13. In this case, the curves for

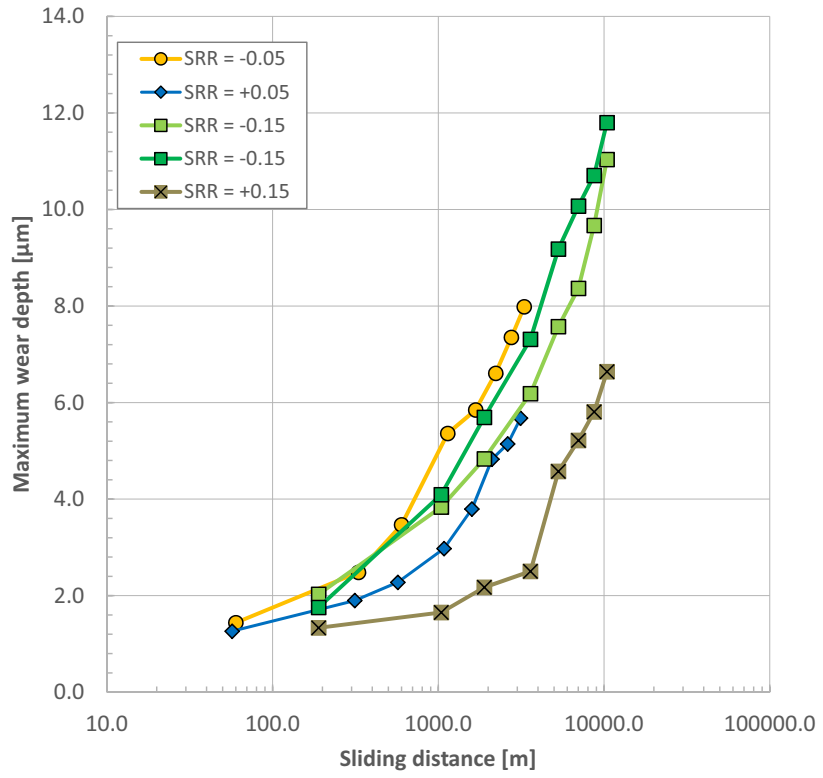

Fig. 16 Comparison of average maximum micropitting wear depths measured at positive and negative slide-roll ratios of \pm 0.05 and \pm 0.15 plotted against the sliding distance; $p_{0}=1.78 \mathrm{GPa}$ 
different directions of sliding do not collapse on the same master curve, in contrast to Fig. 13. It is clear from Fig. 16 that despite the fact that the total sliding distance is broadly the same for a given absolute value of SRR regardless of the direction of sliding, the micropitting wear was much higher for negative SRRs than positive ones at a given absolute value of SRR. The difference is particularly apparent in the case of SRR $= \pm 0.15$ since at this level of SRR the amount of micropitting is comparatively high, so relative differences become more apparent. This observation suggests the presence of additional mechanisms contributing to damage in the case of negative SRR as will be discussed later, but here it is worth noting that in gear applications, micropitting often occurs preferentially in the dedendum where the SRR is negative $[1,17]$, although the exact spread of damage in any particular case depends on the balance of other local conditions such as contact pressure, lambda ratio and mild wear coefficient.

As a summary of the effect of SRR on the extent of micropitting damage, Fig. 17 shows the final values of the maximum micropitting wear depth for all magnitudes and directions of SRR studied here. A clear trend is evident where the level of damage is highest at the largest negative $\mathrm{SRR}, \mathrm{SRR}=-0.3$, and steadily decreases as the SRR reduces to -0.15 and -0.05 and then decreases further as SRR becomes positive at 0.05 .

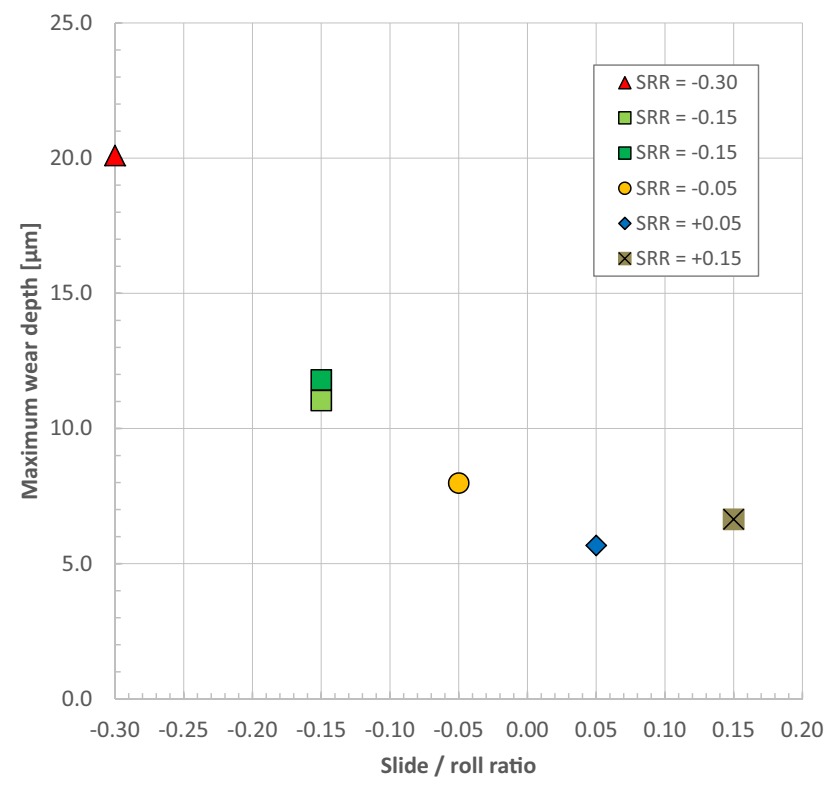

Fig. 17 Plot of maximum wear depth on the roller measured at 5.12 million contact cycles against slide-roll ratio at $1.78 \mathrm{GPa}$

\subsection{Influence of Hertz Pressure on Progression of Micropitting}

The effect of applied Hertz pressure on micropitting damage at a fixed slide-roll ratio was also briefly studied. Figure 18 plots the average maximum wear depth at $S R R=-0.15$ for 1.78 GPa and 1.4 GPa maximum Hertzian contact pressures. As may intuitively be expected given the fatigue origins of micropitting damage, reducing contact pressure from 1.78 to $1.4 \mathrm{GPa}$ has the effect of significantly decreasing micropitting severity.

\section{Discussion}

The micropitting test results clearly showed that increasing the slide-roll ratio at a fixed entrainment speed, i.e. increasing the magnitude of sliding velocity, increases the severity of micropitting damage. In addition, the level of damage correlated very well with the sliding distance covered by the roller at different slide-roll ratios. The latter observation suggests that it is not the magnitude of the SRR itself that drives the increase in damage but that other mechanisms related to the sliding distance are at play. The experiments that led to these observations were performed under closely controlled contact conditions so that the influence of SRR itself was isolated. In particular, increasing the SRR can lead to an increase in bulk (oil inlet) temperature and the associated reduction in EHL film thickness; this effect was eliminated in the tests by adjusting the oil sump temperature in

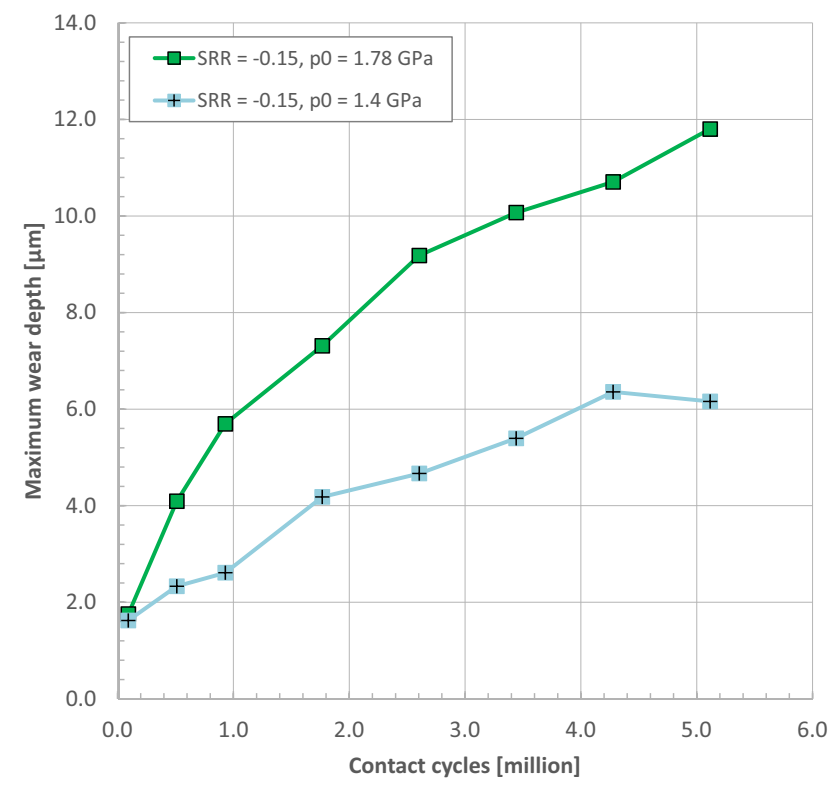

Fig. 18 Average maximum wear depth at $\mathrm{SRR}=-0.15$ for $1.78 \mathrm{GPa}$ and 1.4 GPa maximum Hertz contact pressure 
order to keep the oil inlet temperature the same for all SRRs tested. The increased micropitting damage at higher SRRs is therefore not caused by any reduction in film thickness but by some other mechanism(s). These will be discussed now.

\subsection{Effect of Slide-Roll Ratio on EHL Film Thickness}

One recently proposed mechanism to explain how sliding affects micropitting damage is that the increase in SRR leads to an increase of in-contact temperature which is then suggested to cause a drop in elastohydrodynamic film thickness and thus produce more micropitting [20, 21, 40]. In recent years, this mechanism has been subject of a wider discussion as part of efforts by international gear standard bodies to produce a way of assessing micropitting risk in gear applications $[18,19]$. Although it is well established that in-contact temperature does indeed rise with increasing sliding velocity (see for example [22, 31]), the established EHL theories show that this only affects contact friction through the associated drop of in-contact oil viscosity, but has no effect on film thickness because this is determined by the lubricant properties, and hence the temperature, in the inlet to the contact. Several authoritative works exist discussing the relative effects of in-contact oil properties and inlet oil properties on EHL film thickness and traction; Ref. [22] for example gives a good overview. Review of literature also reveals experimental studies where SRR in the range from pure rolling to $100 \%$ was shown not to affect EHL film thickness, see for example [41, 42]. Although one may expect that at SRR magnitudes close to pure sliding conditions $(\mathrm{SRR}=200 \%)$ some inlet heating may occur which can lead to a drop of EHL film thickness, evidence suggests that within the SRR range applicable to gears, $(\sim 0-50 \%)$, its effect on the EHL film thickness is negligible. Therefore, the conjecture that SRR increases micropitting via an associated decrease in EHL film thickness warrants further scrutiny. What is not in doubt is the clear correlation between higher SRR and higher micropitting damage; the question that needs answering here is whether this correlation implies direct causation or there is a third factor at play. To study this further, the effect of slide-roll ratio on elastohydrodynamic film thickness was investigated here by performing EHL film thickness measurements over a range of SRRs.

These measurements were performed on a PCS Instruments EHD 2 Rig [43] which employs a spacer layer optical interferometry technique to measure oil films with accuracy of a few nm $[37,44]$. The rig employs a ball-on-disc configuration as standard; however, in this study additional tests were performed with a barrel roller on a disc set-up since this more closely reproduces the elliptical contact geometry that was employed in the micropitting tests. The barrel roller major to minor radius ratio was 7 to 1 and contact was formed with the major axis transverse to rolling as is the case in the micropitting tests. In this test set-up the contact is formed between a ball (or a roller) and a disc within a temperature-controlled oil bath so that no significant bulk heating effects occur. The disc and the ball (or roller) are driven by separate motors so that any SRR can be achieved.

Figure 19 shows the measured central film thickness values for the elliptical contact geometry for two different loads $(20 \mathrm{~N}$ and $50 \mathrm{~N}$ ) and SRRs ranging from 0 (pure rolling) to $100 \%$ (where $200 \%$ is pure sliding). This range of SRR more than covers the conditions pertinent to machine elements where micropitting may occur, such as bearings and gears. It is evident that within this range of conditions the SRR has no effect on the central film thickness. The effect of load is also minimal as may be expected from the EHD theory. In order to investigate the effects of SRR on the distribution of the film thickness over the whole contact, rather than just the central film thickness, 3D film thickness maps were produced on the same rig using the standard spacer layer imaging method (SLIM) [44] and the standard ball-on-disc configuration. Figure 20 shows the resulting three-dimensional film thickness maps for $\mathrm{SRR}=0,50,100$ and $180 \%$. It can be seen that the film shape remains generally unaffected by the increase of SRR. In particular, the central film region is almost identical in all cases. Figure 21 affords a closer look at the relevant trends. This shows two-dimensional film thickness profiles parallel to rolling direction extracted from the 3D SLIM measurements for a range of SRR. The profiles were obtained by selecting a single line through the centre of the contact. It can be seen that over the range of slide-roll ratios most relevant to gear contacts, i.e. 0-50\%, both the minimum and central film thickness values do not change with increasing SRR. Beyond 100\% SRR, there is a slight decrease $(<10 \%)$ in central and minimum film thickness values. This is likely to be due to inlet heating which can be significant at such high SRR, but is in any case irrelevant in the context of micropitting damage as the drop is

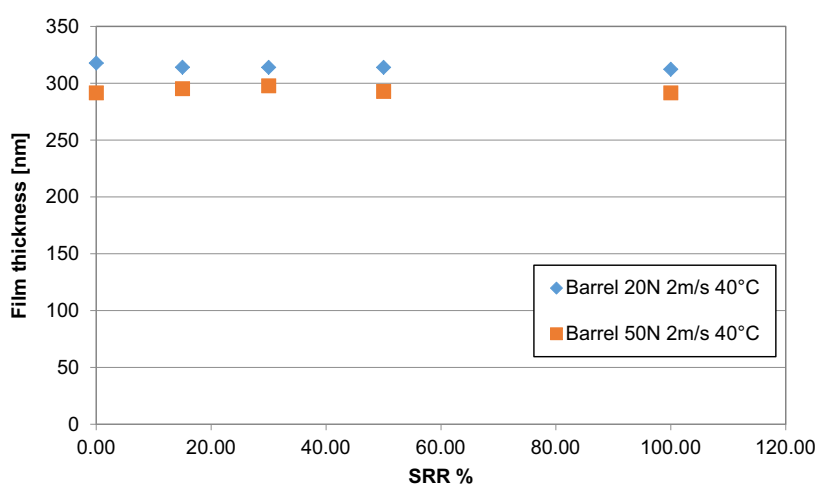

Fig. 19 Variation of central elastohydrodynamic film thickness with slide-roll ratio measured with barrel on disc specimen configuration at two different loads. The major axis of the employed elliptical contact was transverse to rolling direction 

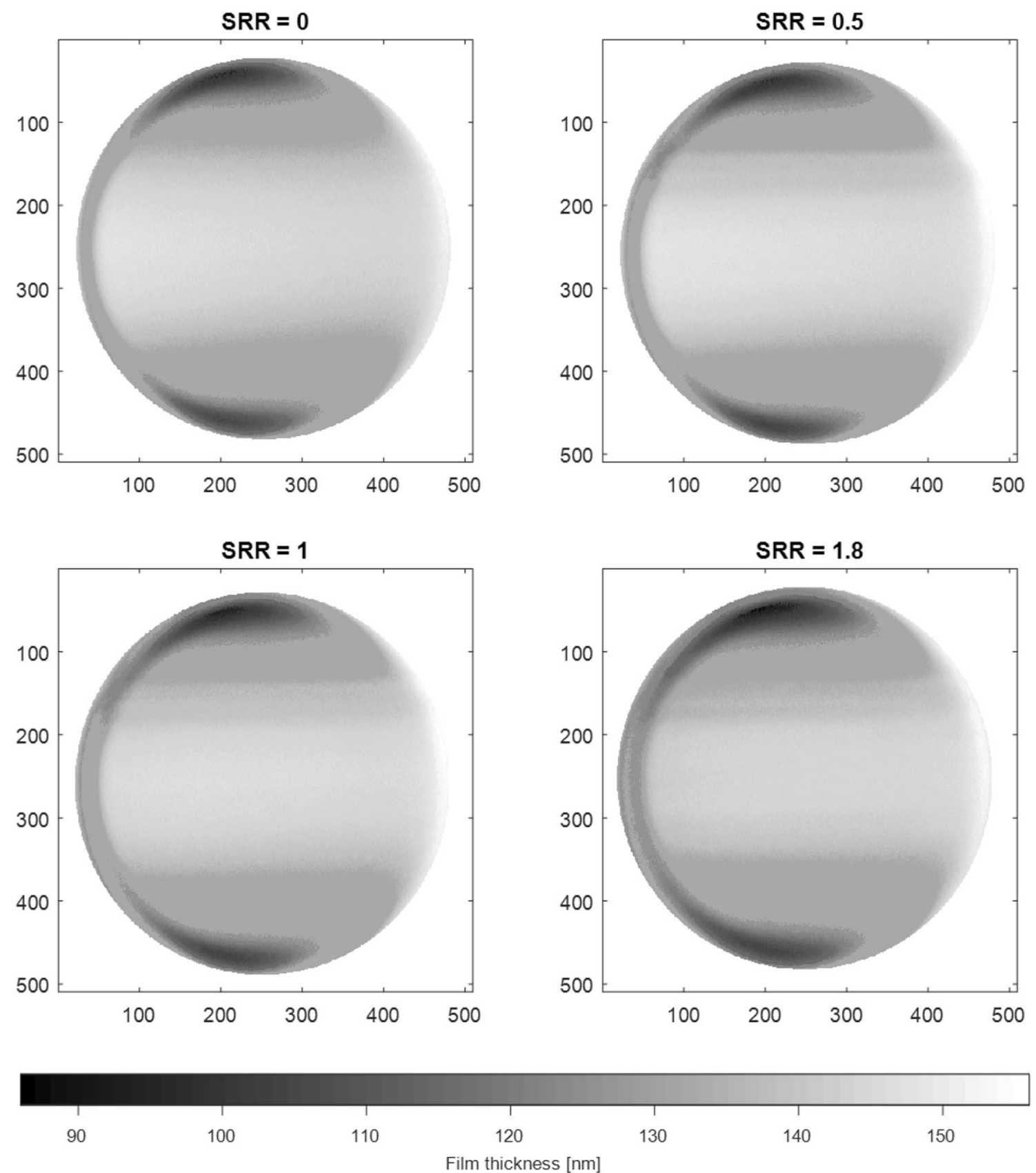

Fig. 20 Three-dimensional maps of elastohydrodynamic film thickness measured using a ball-on-disc specimen configuration at different levels of slide-roll ratio

relatively small and occurs at SRR levels much higher than those encountered in machine components where micropitting is a problem.

The EHL film thickness measurements presented here clearly show that the higher amount of micropitting damage observed at higher slide-roll ratios is not caused by a reduction of EHL film thickness with increasing slide-roll ratio. Furthermore, the obvious influence of the direction of sliding on the extent of micropitting at the same absolute value of SRR clearly cannot be caused by any EHL film thickness variations since the entrainment speed and total frictional heat input are the same regardless of the direction of sliding. In fact, notwithstanding the evidence presented here, given that micropitting generally occurs at very low specific film thicknesses ( $\Lambda$ ratios), it is difficult to see how any small variations in the calculated values of this already low $\Lambda$ value can have a major effect on progression of micropitting. 


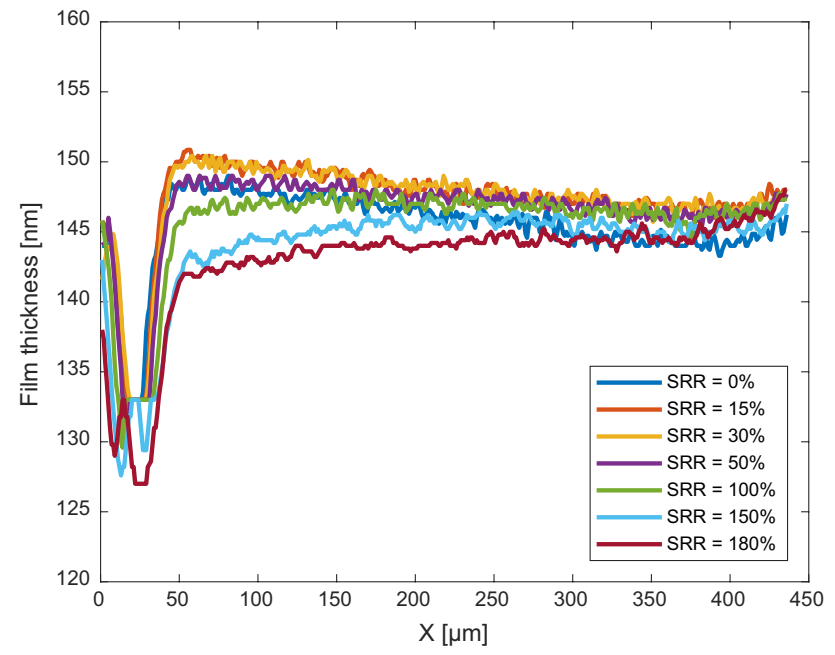

Fig. 21 Elastohydrodynamic film thickness profiles across the centre of contact in the direction of rolling measured at different levels of slide-roll ratio in a ball-on-disc configuration

\subsection{Consideration of Local Asperity Contact Conditions}

The micropitting experiments presented here were designed to isolate the influence of SRR on micropitting by keeping the EHL film thickness and contact friction the same. Therefore, the observed increase in micropitting damage with increasing SRR is not caused by variation of EHL film or friction on macro-contact level. Given that the primary cause of micropitting are the local, asperity-level stresses, it is necessary to investigate how the conditions on this local asperity level may be influenced by changing SRR.

Micropitting is ultimately caused by the mechanism of contact fatigue on the asperity level. This requires a fatigue crack to be formed and then propagated under the action of normal and tangential stresses acting on the asperity microcontacts, in much the same way that pitting damage is caused by a fatigue crack on the macro-contact level. Initiation and progression of such contact fatigue damage is known to be heavily influenced by the magnitude of stresses and the number of stress cycles, as reflected in standard bearing fatigue life equations [45] and discussed at length in the literature, for example [1, 25, 46, 47]. In lubricated, rough surface contacts, asperity interactions and normal and tangential stresses in asperity micro-contacts are difficult to predict or measure. Rough surface dry contact models clearly show that local stress fields are heavily influenced by the geometry of asperities, with local shear stress magnitudes being related to asperity slopes and their depths to roughness correlation length or similar measures of asperity spatial distribution [48]. These general trends remain valid under lubricated conditions when specific film thicknesses are low so that significant asperity-to-asperity contact occurs, which in turn suggests that the lambda ratio itself is not a sufficient measure of the risk of micropitting but that the actual structure of roughness matters too. Under lubricated conditions, even at low lambda ratios, additional effects are present at asperity micro-contacts not least due to micro-EHL films. Factors governing the micro-EHL films are not well understood but some aspects of their influence on normal stresses and friction in asperity contacts have been explored in the literature, including the potential effects of sliding which may therefore be relevant to the observations presented in this paper. For example, Olver et al. [49] have shown that sliding may increase asperity stresses under mixed lubrication conditions: under pure rolling, the presence of lubricant seems to provide a 'cushioning' effect so that the asperity pressures are somewhat lower than those that may be found in an equivalent unlubricated contact; however, when sliding is introduced this cushioning effect seems to disappear and the asperity pressures return to the levels similar to those found in the equivalent dry contact. Given the strong effect of stress magnitude on fatigue lives, this increase in local asperity pressures may well contribute to increased micropitting damage when in sliding-rolling as opposed to pure rolling contacts. However, the authors only studied one SRR $(62 \%)$ so that it is not possible to say at this time whether this effect may also be partly responsible for the increase in micropitting damage with increasing SRR. Guegan et al. $[41,42]$ did not observe any influence of the SRR on the local film thickness in rough surface contacts operating under mixed lubrication conditions and also showed that the contribution of roughness to the overall friction under these conditions was not affected by the SRR, with any increase in friction at higher SRR being the same as may be expected with ideally smooth contacts. They did, however, observe other micro-EHL-related phenomena that may affect micropitting performance: they showed that with increasing entrainment speed, the asperity pressures near the periphery of the contact may decrease, while those in the central region of the contact increase, which may lead to increased fatigue damage locally; they also observed that micro-EHL contacts provide a significant contribution to overall contact friction even after complete 'lift-off' is achieved, i.e. after all asperities are separated by micro-EHL films. This suggests that friction behaviour on the local asperity level is more complex than simply being equal to the boundary friction coefficient on macro-level as is often assumed in theoretical treatment of mixed lubrication. Given the known influence of friction on rolling contact fatigue lives [50], these aspects of frictional behaviour of micro-EHL contacts may be of significance to the micropitting performance of a given contact.

Given the fatigue origins of micropitting, in addition to the implications of the magnitude of normal and tangential stresses on local asperities discussed above, the number of 
stress cycles experienced by asperities is also of significance. In this respect, it is possible to establish the influence of SRR with a relatively high certainty. This can be done by solving the relevant rough surface contact problem at different time steps to produce a contact stress history for each asperity as the two bodies roll and slide over each other, along the lines employed in [51]. To this aim, the present study employs an existing numerical dry contact model, originally devised by Webster and Sayles [52] and later extended by Sayles and co-authors [34, 53, 54], to predict contact pressures in the roller-disc contacts of the MPR test rig at the employed loading conditions and using the real measured surface roughness profiles. The effect of SRR on the stress history of a select single asperity in the contact can then be established by plotting the variation of the normal stress experienced by the chosen point as it travels through the contact at the particular sliding speed of interest. The use of a dry contact model can be justified here on the basis that tests were conducted at very low $\Lambda$ ratio of 0.1 so that solid-to-solid contact dominates and the fact that only the general trends in the number of stress cycles are of interest here rather than the exact magnitudes of asperity stresses. As an example, Fig. 22 shows a map of surface pressures thus calculated for a single instant in time. Figure 23 then shows the normal stress histories for two random points on the roller surface as they travel through the contact with the counterface disc. For comparison purposes stress histories for five different slide-roll ratios are shown, ranging from pure rolling to $\mathrm{SRR}=90 \%$. In these examples, the time taken for a surface point to travel across the contact width in rolling direction is split into 60 equal time steps. What is immediately apparent in this figure is that an increase in SRR results in an increased number of asperity stress cycles as indicted by more frequent stress oscillations as SRR increases. The curves for pure rolling $(S R R=0 \%)$ show a smooth stress history with a single stress cycle, since the point under consideration will remain in contact with the same point on the disc surface throughout its passage through the contact. On the other hand, the introduction of sliding causes an asperity on the roller to come in and out of contact with several opposing asperities on the counterface disc during its passage through the contact, so that it now experiences a number of stress cycles. At a fixed rolling speed, the higher the slide-roll ratio the higher the sliding speed and hence the more stress cycles any given asperity will experience. This is particularly apparent in the figure for SRR of $30 \%$ and $90 \%$, the latter deliberately included here as a hypothetical example to clearly illustrate the described effect. The actual magnitude of the normal stress for the two example points used here is seen to vary between zero (point not in contact with any other point on the opposing surface) and about $3 \mathrm{GPa}$. This compares with the maximum Hertz pressure of $1.78 \mathrm{GPa}$ employed in the tests.

Given that micropitting is caused by rolling contact fatigue, clearly an increased number of stress cycles would result in higher fatigue damage and therefore an increased severity of micropitting. Consequently, the results of Fig. 23 go some way in explaining the mechanism by which the increased levels of sliding produce a higher amount of micropitting; namely, with all other things being equal, an increase in slide-roll ratio increases the number of asperity stress cycles, which promotes contact fatigue on the asperity level and hence results in more micropitting. This mechanism is also supported by the fact that the micropitting damage progression was seen to correlate well with the sliding distance regardless of the SRR. For a given roughness topography of the two surfaces and a fixed entrainment speed, the number of asperity stress cycles, and hence the level of accumulated fatigue damage, is proportional to the sliding distance and, consequently, the same severity of micropitting damage would be expected to occur for the same sliding distance regardless of the SRR. Although other micro-EHL effects may be taking place in asperity contacts as described earlier, the fact that the observed damage so clearly correlates with the sliding distance at different SRRs suggests that the number of asperity stress cycles is the main mechanism by which the SRR affects micropitting.

These findings indicate that any design parameter intended to assess the risk of micropitting in practical applications should account for asperity stress history. Given the inherently transient nature of local stress conditions in a rough surface contact where roughness evolves continuously, be it through micropitting itself or mild wear, it is not trivial to achieve this in any kind of deterministic manner. However, a good attempt can be made by using appropriate numerical models of mixed lubrication that include surface roughness evolution, asperity stress history and fatigue damage accumulation, such as those in [8,13-15] for example. Such models do not currently account for crack propagation and present results show that this should be addressed if more accurate predictions of
Fig. 22 An example map of the calculated contact pressures for the disc-roller contact at a given instant in time

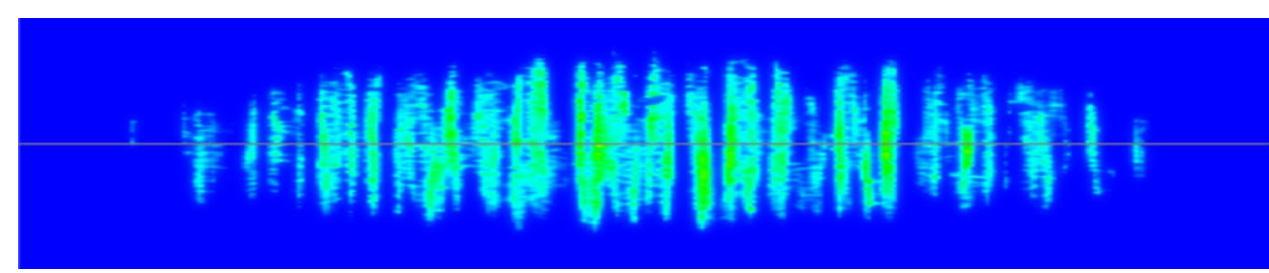


Fig. 23 Stress histories for two selected points on the roller surface during their passage through a roller-disc contact at different slide-roll ratios and at loading conditions used in the tests (Note: The $\mathrm{x}$-axis label 'Time step ID' denotes the instant in time when the given stress is acting on the point being followed; the time sampling interval is uniform in all cases)
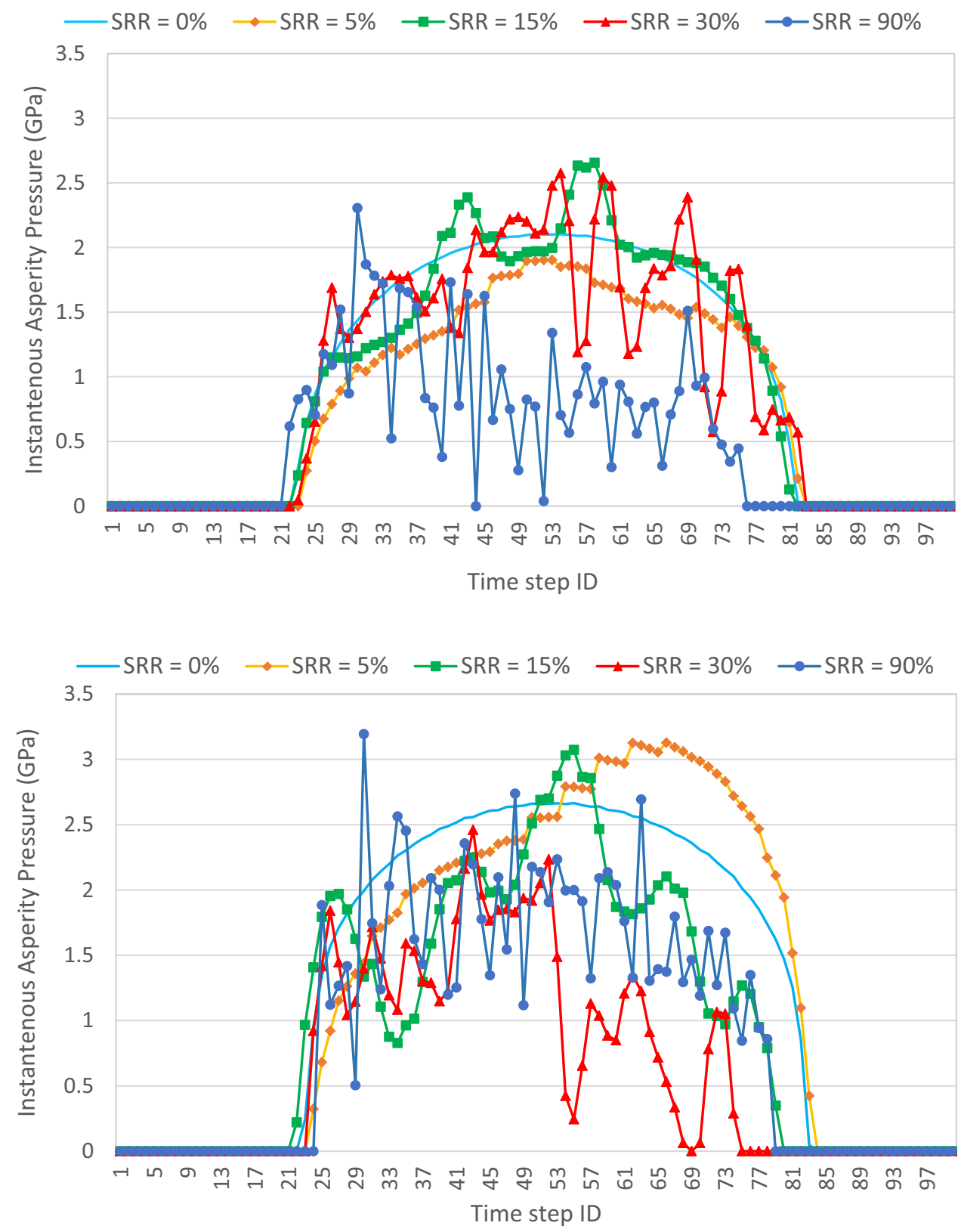

micropitting damage progression are to be achieved. In particular, it was seen here that the direction of sliding has a very important influence on micropitting and this is likely to be through its effect on crack propagation rather than initiation. The influence of sliding direction on surface fatigue damage has been suggested to be due to the enhanced crack growth caused by pressurization of lubricant trapped inside a surfacebreaking crack under negative sliding $[38,39]$, so to account for it, a model would need to consider crack propagation and not just initiation. In any case, for the purposes of gear design, a much simpler micropitting criterion is required if it is to be widely accepted. While the difficulties in devising such a simple criterion that is able to account for the wide range of influencing factors discussed here are acknowledged, one potential way forward would be to use the existing numerical models to produce approximate design charts which may then be validated through suitable experiments. Such charts should include not only the gear loading and speed parameters, but also the gear macro geometry and surface finish in order to account for the local aperity stress history during tooth meshing.

\section{Conclusion}

This study investigated the progression of micropitting damage in rolling-sliding non-conformal contacts under conditions pertinent to gear applications, with particular 
focus on the influence of slide-roll ratio (SRR). The devised test methodology employs a triple-disc rolling contact fatigue rig which allows the influence of the SRR to be isolated through a close control of contact conditions. In particular, any variation in bulk temperature at different SRR, and its subsequent influence on EHL film thickness and friction, was eliminated by considering the thermal network model of the triple-disc set-up and adjusting the oil-sump temperature accordingly. This allowed for the influence of the magnitude of sliding to be ascertained at the same specific oil film thickness ( $\Lambda$ ratio). Potential influence of SRR on EHL film thickness is studied in parallel using an optical interferometry film thickness rig. Finally, an existing numerical model for rough surface contacts is used to study the stress histories experienced by contacting asperities at different SRRs. The experimental observations are then discussed in view of these local, asperity-level stress conditions. The main findings can be summarized as follows:

- Increasing the slide-roll ratio at a fixed entrainment speed leads to an increased level of micropitting damage. The amount of micropitting damage correlates well with sliding distance regardless of the magnitude of the slide-roll ratio.

- Negative slide-roll ratios (i.e. when the component of interest is slower than the counterface) lead to more micropitting damage than the equivalent positive SRRs. This supports the postulate that propagation rates of surface-breaking cracks under negative sliding are enhanced.

- In the absence of bulk heating, no reduction in the value of central or minimum EHL film thickness is observed with increasing slide-roll ratio in the range $S R R=0$ to $100 \%$. Therefore, the postulate made in recent literature that the increase in SRR causes more micropitting through the reduction of EHL film thickness and the proposed micropitting risk criterion based on this postulate are not supported by the present results.

- Analyses of asperity stress history as the surfaces slide past each other, using an established numerical model of rough surface contacts, show that roughness asperities experience a greater number of micro-contact stress cycles as the slide-roll ratio increases. Given the fatigue origins of micropitting damage, and therefore its dependence on the number of stress cycles, this means that the main mechanism by which increasing SRR leads to increased micropitting damage is by increasing the number of asperity stress cycles. Since the number of asperity stress cycles is proportional to the sliding distance, this mechanism is supported by the fact that the amount of micropitting damage was observed here to correlate with sliding distance regardless of the actual SRR.

- These findings indicate that any potential design criteria intended to assess the risk of micropitting in real machine elements, such as gears, need to account for the time history of local asperity stresses for given operating conditions and surface finish. One potential way to achieve this is to utilize numerical models of rough contacts to generate design charts which indicate the risk of micropitting in terms of gear geometry, surface finish and operating parameters.

Open Access This article is distributed under the terms of the Creative Commons Attribution 4.0 International License (http://creativeco mmons.org/licenses/by/4.0/), which permits unrestricted use, distribution, and reproduction in any medium, provided you give appropriate credit to the original author(s) and the source, provide a link to the Creative Commons license, and indicate if changes were made.

\section{References}

1. Olver, A.V.: The mechanism of rolling contact fatigue: an update. Proc. Inst. Mech. Eng. J 219, 313-330 (2005)

2. Dawson, P.H.: Effect Of metallic contact on the pitting of lubricated rolling surfaces. Mech. Eng. Sci. 4(1), 16-21 (1962)

3. Dawson, P.H.: Further experiments on the effect of metallic contact on the pitting of lubricated rolling surfaces. Proc. Inst. Mech. Eng. 180, 95-100 (1965)

4. Benyajati, C., Olver, A.V., Hamer, C.J.: An experimental study of micropitting, using a new miniature test-rig. In: Dalmaz, G., Lubrecht, A.A., Dowson, D., Priest, M. (eds.) Transient Processes in Tribology, pp. 601-610. Elsevier, Lyon (2003)

5. Lainé, E., Olver, A.V., Beveridge, T.A.: Effect of lubricants on micropitting and wear. Tribol. Int. 41, 1049-1055 (2008)

6. Webster, M.N., Norbart, C.J.J.: An experimental investigation of micropitting using a roller disk machine. Tribol. Trans. 38, 883-893 (1995)

7. Oila, A., Bull, S.J.: Assessment of the factors influencing micropitting in rolling/sliding contacts. Wear 258, 1510-1524 (2005)

8. Evans, H.P., Snidle, R.W., Sharif, K.J., Shaw, B.A., Zhang, J.: Analysis of micro-elastohydrodynamic lubrication and prediction of surface fatigue damage in micropitting tests on helical gears. J. Tribol. 135, 011501 (2012)

9. Clarke, A., Weeks, I.J.J., Snidle, R.W., Evans, H.P.: Running-in and micropitting behaviour of steel surfaces under mixed lubrication conditions. Tribol. Int. 101, 59-68 (2016)

10. Cen, H., Morina, A., Neville, A.: Effect of slide to roll ratio on the micropitting behaviour in rolling-sliding contacts lubricated with ZDDP-containing lubricants. Tribol. Int. 122, 210-217 (2018)

11. Kadiric, A., Rycerz, P.: Influence of contact conditions on the onset of micropitting in rolling-sliding contacts pertinent to gear applications. In: AGMA Fall Technical Meeting 2016, 16FTM21 (2016)

12. Vrcek, A., Hultqvist, T., Baubet, Y., Björling, M., Marklund, P., Larsson, R.: Micro-pitting and wear assessment of engine oils operating under boundary lubrication conditions. Tribol. Int. 129, 338-346 (2019) 
13. Brandão, J.A., Seabra, J.H.O., Castro, J.: Surface initiated tooth flank damage. Part II: prediction of micropitting initiation and mass loss. Wear 268, 13-22 (2010)

14. Li, S., Kahraman, A.: A micro-pitting model for spur gear contacts. Int. J. Fatigue 59, 224-233 (2014)

15. Morales-Espejel, G.E., Rycerz, P., Kadiric, A.: Prediction of micropitting damage in gear teeth contacts considering the concurrent effects of surface fatigue and mild wear. Wear 398-399, 99-115 (2018)

16. Lainé, E., Olver, A.V., Lekstrom, M.F., Shollock, B.A., Beveridge, T.A., Hua, D.Y.: The effect of a friction modifier additive on micropitting. Tribol. Trans. 52, 526-533 (2009)

17. Errichello, R.: Morphology of micropitting. Gear Technol. 4, 74-81 (2012)

18. Publication, B.S.I.S.: ISO/TR 15144-1:2014: Calculation of micropitting load capacity of cylindrical spur and helical gearsPart 1: Introduction and basic principles. (2014)

19. ISO/TS 6336-22:2018 Calculation of load capacity of spur and helical gears - Part 22: Calculation of micropitting load capacity. (2018)

20. Kissling, U.: The application of the first international calculation method for micropitting. In AGMA Fall Technical Meeting 2011. (2011)

21. Kissling, U.: International calculation method for micropitting. Gear Solut. 2012, 47-54 (2012)

22. Spikes, H.A., Zhang, J.: History, origins and prediction of elastohydrodynamic friction. Tribol. Lett. 56, 1-25 (2014)

23. Gohar, R.: Elastohydrodynamics. World Scientific, Singapore (2001)

24. Graham, R.: Pitting of Hard Steel Rolling-Sliding Elastohydrodynamically Lubricated Line Contacts, PhD Thesis, Imperial College London (1979)

25. Rycerz, P., Olver, A., Kadiric, A.: Propagation of surface initiated rolling contact fatigue cracks in bearing steel. Int. J. Fatigue 97, 29-38 (2017)

26. Manieri, F., Stadler, K., Morales-Espejel, G., Kadiric, A.: The origins and significance of white etching cracks in bearing steels. Int. J. Fatigue 120, 107-133 (2019)

27. Olver, A. V.: Wear of Hard Steel in Rolling Contact, PhD Thesis, Imperial College London (1986)

28. Olver, A.V.: Testing transmission lubricants: the importance of thermal response. Proc. Inst. Mech. Eng. G 205, 35-44 (1991)

29. Hajishafiee, A., Kadiric, A., Ioannides, S., Dini, D.: A coupled finite-volume CFD solver for two-dimensional elasto-hydrodynamic lubrication problems with particular application to rolling element bearings. Tribol. Int. 109, 258-273 (2017)

30. AGMA 925-A03: Effect of Lubrication on Gear Surface Distress

31. Olver, A.V., Spikes, H.A.: Prediction of traction in elastohydrodynamic lubrication. Proc. Inst. Mech. Eng. J 212, 321-332 (1998)

32. Kadiric, A., Christodoulias, A., Olver, A., Sworski, A., Kolekar, A., Lockwood, F.: The efficiency of a simple Spur Gearbox-thermally coupled lubrication model. AGMA Tech. Pap. 14FTM08 2014, 81-98 (2014)

33. Jaeger, J.: Moving sources of heat and the temperature of sliding contacts. J. Proc. R. Soc. New South Wales 76, 203-224 (1942)

34. Kadiric, A., Sayles, R.S., Ioannides, E.: Thermo-mechanical model for moving layered rough surface contacts. J. Tribol. 130, $011016(2008)$

35. Blok, H.: Theoretical study of temperature rise of surfaces of actual contact under oiliness lubricating conditions. In:
Proceedings of General Discussion on Lubrication, Part 2, (Institution of Mechanical Engineers, London). p. 222 (1937)

36. Hamrock, B.J., Dowson, D.: Ball Bearing Lubrication: Elastohydrodynamics of Elliptical Contacts. Wiley, California (1981)

37. Cann, P.M., Spikes, H.A., Hutchinson, J.: The development of a spacer layer imaging method (SLIM) for mapping elastohydrodynamic contacts. Tribol. Trans. 39, 915-921 (1996)

38. Kaneta, M., Yatsuzuka, H., Murakami, Y.: Mechanism of crack growth in lubricated rolling/sliding contact. A S L E Trans. 28, 407-414 (1985)

39. Bower, A.F.: The influence of crack face friction and trapped fluid on surface initiated rolling contact fatigue cracks. J. Tribol. 110, 704-711 (1988)

40. Elstorpff, M.G.: Einflusse auf die Grubchentragfahigkeit ennsatzgeharterer Sahnrader bis in das hochste Zeitfestigkeitsgebiet. PhD Thesis, Technische Universität München (1993)

41. Guegan, J., Kadiric, A., Spikes, H.: A study of the lubrication of EHL point contact in the presence of longitudinal roughness. Tribol. Lett. 59, 22 (2015)

42. Guegan, J., Kadiric, A., Gabelli, A., Spikes, H.: The relationship between friction and film thickness in EHD point contacts in the presence of longitudinal roughness. Tribol. Lett. 64, 33 (2016)

43. PCS EHD 2 Rig Description, https://pcs-instruments.com/product/ ehd-elasto-hydrodynamic-lubriction-2/. Accessed 19 April 2019

44. Spikes, H.A., Cann, P.M.: The development and application of the spacer layer imaging method for measuring lubricant film thickness. Proc. Inst. Mech. Eng. J. 215, 261-277 (2001)

45. ISO: ISO 281:2007: "Rolling Bearings—Dynamic Load Ratings and Rating Life." (2008)

46. Morales-Espejel, G.E., Gabelli, A., de Vries, A.J.C.: A model for rolling bearing life with surface and subsurface survival-tribological effects. Tribol. Trans. 58, 894-906 (2015)

47. Ioannides, E., Harris, T.A.: A new fatigue life model for rolling bearings. J. Tribol. 107, 367-377 (1985)

48. Kadiric, A., Sayles, R.S., Zhou, X.B., Ioannides, E.: A numerical study of the contact mechanics and sub-surface stress effects experienced over a range of machined surface coatings in rough surface contacts. J. Tribol. 125, 720 (2003)

49. Olver, A.V., Guangteng, G., Spikes, H.A.: Film thickness and pressure in micro-EHD contacts. Tribol. Ser. 38, 503-510 (2000)

50. Diaconescu, E.: Pitting Under Traction In Heavily Loaded Point Contacts, PhD Thesis, Imperial College London (1975)

51. Kim, T.H., Olver, A.V.: Stress history in rolling-sliding contact of rough surfaces. Tribol. Int. 31, 727-736 (1998)

52. Webster, M.N., Sayles, R.S.: A numerical model for the elastic frictionless contact of real rough surfaces. J. Tribol. 108, 314-320 (1986)

53. Nyqvist, J., Kadiric, A., Ioannides, S., Sayles, R.: Semi-analytical model for rough multilayered contacts. Tribol. Int. 87, 98-112 (2015)

54. West, M.A., Sayles, R.S.: A 3-dimensional method of studying 3-body contact geometry and stress on real rough surfaces. In: Dowson, D., Taylor, C.M., Godet, M., Berthe, D. (eds.) Interface Dynamics, vol. 12, pp. 195-200. Elsevier, Amsterdam (1987)

Publisher's Note Springer Nature remains neutral with regard to jurisdictional claims in published maps and institutional affiliations. 